\title{
1 Prolonged $\beta$-Adrenergic Stimulation Disperses Ryanodine Receptor Clusters in Cardiomyocytes: Implications for Heart Failure
}

3

\author{
Xin Shen ${ }^{1,2}$, Jonas van den Brink ${ }^{3}$, Anna Bergan-Dahl ${ }^{1,2}$, Terje R. Kolstad ${ }^{1,2}$, \\ Einar S. Norden ${ }^{1,2}$, Yufeng Hou ${ }^{1,2}$, Martin Laasmaa ${ }^{1,2}$, Ann P. Quick ${ }^{4,5}$, Emil K.S. Espe ${ }^{1,2}$, Ivar \\ Sjaastad $^{1,2}$, Xander H.T. Wehrens ${ }^{4,5}$, Andrew G. Edwards ${ }^{6}$, Christian Soeller ${ }^{7}$, \\ William E. Louch ${ }^{1,2}$ \\ ${ }^{1}$ Institute for Experimental Medical Research, Oslo University Hospital and University of Oslo, NO-0424 Oslo, \\ Norway \\ ${ }^{2}$ K.G. Jebsen Centre for Cardiac Research, University of Oslo, Oslo Norway \\ ${ }^{3}$ Simula Research Laboratory, Lysaker, Norway \\ ${ }^{4}$ Department of Molecular Physiology \& Biophysics, Cardiovascular Research Institute, Baylor College of \\ Medicine, Houston, TX, USA \\ ${ }^{5}$ Section of Cardiology, Departments of Medicine and Pediatrics, Baylor College of Medicine, Houston, TX, \\ USA \\ ${ }^{6}$ Department of Pharmacology, UC Davis, Davis, California, USA \\ ${ }^{7}$ Biomedical Physics, University of Exeter, Exeter, UK
}

Short title: RyR2 dispersion during $\beta$-Adrenergic stimulation

Manuscript category: Research Reports - Direct Submissions

\section{Corresponding authors:}

Xin Shen, email: xin.shen@medisin.uio.no

William E. Louch, email: w.e.louch@medisin.uio.no

Institute for Experimental Medical Research (IEMR), Oslo University Hospital, Ullevål

PB 4956 Nydalen, NO-0424 Oslo, Norway

Phone: +47230168 00; Fax: +4723016799

\section{Significance statement}

The heartbeat is triggered by the release of $\mathrm{Ca}^{2+}$ from Ryanodine Receptors (RyRs) within cardiomyocytes. Recent data indicate RyR arrangement is highly malleable. However, mechanisms controlling RyR reorganisation and the subsequent impact on $\mathrm{Ca}^{2+}$ homeostasis remain unclear. Here, we show that prolonged $\beta$-adrenergic stimulation causes RyR clusters to disperse, drastically altering the frequency and kinetics of $\mathrm{Ca}^{2+}$ release events called " $\mathrm{Ca}^{2+}$ sparks" in a process that is dependent on CaMKII and PKA. In healthy cells, these compensatory effects protect against arrhythmogenic $\mathrm{Ca}^{2+}$ over-activity. However, during heart failure, RyR hyper-phosphorylation and dispersion impairs $\mathrm{Ca}^{2+}$ release and cardiac performance. Thus, RyR localization and function are intimately linked via channel phosphorylation which, while finely tuned in health, underlies impaired cardiac function during pathology. 


\section{Abstract}

49 Ryanodine Receptors (RyRs) exhibit dynamic arrangements in cardiomyocytes, and we previously showed that

50 "dispersion" of RyR clusters disrupts $\mathrm{Ca}^{2+}$ homeostasis during heart failure (HF) (Kolstad et al., eLife, 2018).

51 Here, we investigated whether prolonged $\beta$-adrenergic stimulation, a hallmark of HF, promotes RyR cluster

52 dispersion, and examined the underlying mechanisms. We observed that treatment of healthy rat cardiomyocytes

53 with isoproterenol for 1 hour triggered progressive fragmentation of RyR clusters. Pharmacological inhibition

54 of CaMKII reversed these effects, while cluster dispersion was reproduced by specific activation of CaMKII,

55 and in mice with constitutively active Ser2814-RyR. A similar role of protein kinase A (PKA) in promoting

56 RyR cluster fragmentation was established by employing PKA activation or inhibition. Progressive cluster

57 dispersion was linked to declining $\mathrm{Ca}^{2+}$ spark fidelity and magnitude, and slowed release kinetics from $\mathrm{Ca}^{2+}$

58 propagation between more numerous RyR clusters. In healthy cells, this served to dampen the stimulatory

59 actions of $\beta$-adrenergic stimulation over the longer term, and protect against pro-arrhythmic $\mathrm{Ca}^{2+}$ waves.

60 However, during HF, RyR dispersion was linked to impaired $\mathrm{Ca}^{2+}$ release. Thus, RyR localization and function

61 are intimately linked via channel phosphorylation by both CaMKII and PKA which, while finely tuned in

62 healthy cardiomyocytes, underlies impaired cardiac function during pathology.

\section{Key words}

65 Cardiomyocyte, Ryanodine Receptor, $\mathrm{Ca}^{2+}$ spark, $\beta$-adrenergic stimulation, heart failure

\section{Abbreviations}

$\beta$-adrenergic receptor

70 CaMKII

$\mathrm{Ca}^{2+} /$ calmodulin-dependent kinase II

71 CICR

$\mathrm{Ca}^{2+}$-induced $\mathrm{Ca}^{2+}$ release

72 CRU

$\mathrm{Ca}^{2+}$ release unit

Full duration half maximum

80 RyR

Full width half maximum

Heart failure

L-type $\mathrm{Ca}^{2+}$ channel

Protein kinase A

Ryanodine Receptor type 2

Sarcoplasmic reticulum

Time to $\mathrm{F}_{50}$

Time to peak 


\section{Introduction}

In cardiomyocytes, the processes underlying initiation of contraction are well described, at least at the macroscale. Sarcolemmal depolarisation triggers an influx of $\mathrm{Ca}^{2+}$ through voltage-gated L-type $\mathrm{Ca}^{2+}$ channels (LTCCs) within t-tubules, which in turn elicits a much larger release of $\mathrm{Ca}^{2+}$ into the cytosol via ryanodine receptors (RyRs) in the sarcoplasmic reticulum (SR). This process of $\mathrm{Ca}^{2+}-$ induced $\mathrm{Ca}^{2+}$ release (CICR) depends on the precise organization of LTCCs and RyRs within dyadic junctions of the two membranes, with LTCCs located in nanoscale apposition to RyRs across the dyadic cleft $^{1}$. Intrinsically, RyRs are organized into discrete clusters, and these arrangements are thought to be critical in defining channel function. Indeed, RyR clusters located within close proximity are proposed to cooperatively operate as $\mathrm{Ca}^{2+}$ Release Units (CRUs) to generate $\mathrm{Ca}^{2+}$ sparks $^{2,3}$, the elementary units of $\mathrm{SR} \mathrm{Ca}^{2+}$ release$^{4}$. Recent advances in high-resolution imaging techniques such as $3 \mathrm{D} \mathrm{dSTORM}^{5}$, DNA-PAINT ${ }^{6}$ and electron tomography ${ }^{7}$ have created an opportunity to understand these dyadic arrangements in unprecedented detail.

Contractile dysfunction and arrhythmogenesis are hallmarks of heart failure (HF), and considerable data have linked these phenomena to t-tubule disruption in this condition ${ }^{8-10}$. Recently, we reported that pathological changes occur also on the other side of the dyad, as we observed "dispersion" of RyR clusters in failing cardiomyocytes. This RyR rearrangement specifically included fragmentation of RyR groupings into more numerous, smaller clusters, without any change in overall channel number ${ }^{11}$. RyR dispersion was critically linked to low fidelity spark generation. Furthermore, when $\mathrm{Ca}^{2+}$ release was successfully triggered by a $\mathrm{CRU}$, propagation of $\mathrm{Ca}^{2+}$ between multiple clusters generated sparks with slow kinetics, and the overall $\mathrm{Ca}^{2+}$ transient was desychronized ${ }^{11}$. Similar fragmentation of CRUs has been reported in a sheep model of persistent atrial fibrillation ${ }^{12}$, and in monocrotaline-induced right ventricular failure in rats $^{13}$. Thus, accumulating evidence indicates that RyR mislocalization, and ensuing dysfunction, is a key contributor to pathophysiology.

What drives changes in RyR organization in diseased cardiomyocytes? Previous work has indicated that nanoscale RyR positioning is fine-tuned by phosphorylation of the channels, at least acutely ${ }^{7,14}$, and that both CaMKII- and PKA-dependent phosphorylation of RyR disrupt its function during $\mathrm{HF}^{15-17}$. We presently hypothesized that these two phenomena are intimately linked. We demonstrate that prolonged $\beta$-adrenergic stimulation, as is well known to occur in HF, promotes RyR dispersion via both CaMKIIand PKA-dependent phosphorylation of the channel. This RyR reorganization and sensitization is associated with a time-dependent increase in $\mathrm{Ca}^{2+}$ leak, slowing of $\mathrm{Ca}^{2+}$ spark kinetics, and reduced $\mathrm{Ca}^{2+}$ transient magnitude. In healthy cells, these actions appear to be aimed at gradually countering the stimulatory effects of increased RyR phosphorylation and $\mathrm{Ca}^{2+}$ sensitivity. Indeed, we observed that sufficiently dispersed RyRs inhibit the development of $\mathrm{Ca}^{2+}$ waves which underlie arrhythmia. Conversely, blocking hyper-phosphorylation of RyRs reverses channel dispersion in HF cells and associated impairment of $\mathrm{Ca}^{2+}$ release, providing new insight into the protective mechanisms of $\beta$ blockade in this disease. 
bioRxiv preprint doi: https://doi.org/10.1101/2022.02.18.481024 - this version posted February 19, 2022. The copyright holder for this preprint (which was not certified by peer review) is the author/funder, who has granted bioRxiv a license to display the preprint in perpetuity. It is made available under aCC-BY 4.0 International license.

\section{Results}

\section{Prolonged $\beta$-adrenergic receptor ( $\beta$-AR) activation causes RyR cluster dispersion}

129 Using 3D dSTORM imaging, we investigated the effects of long-term phosphorylation on RyR

130 organization in cardiomyocytes. To this end, we compared RyR arrangements in normal rat ventricular

131 cardiomyocytes to those treated with isoproterenol $(100 \mathrm{nM})$ for varying lengths of time $(10,30$ or 60

$132 \mathrm{~min}$ ). 3D dSTORM reconstructions revealed progressive RyR cluster fragmentation as the duration of

133 isoproterenol application increased (Fig. 1a). Indeed, following a brief, $10 \mathrm{~min}$ bout of isoproterenol

134 exposure, we detected a slight, but non-significant change in measured cluster parameters. However,

135 after 30 minutes of isoproterenol treatment, RyR cluster size significantly decreased (Fig. 1b), while

136 cluster density increased (Fig. 1d). By 1 hour of $\beta$-AR activation, continued RyR rearrangement was

137 sufficient to reduce CRU size, as fewer RyRs remained within clusters located $\leqq 100 \mathrm{~nm}$ from their

138 neighbors (Fig. 1c, see enlargements in Fig. 1a for representative images of CRUs). Since overall RyR

139 density was unchanged by isoproterenol treatment (Fig. 1e), the detected differences in RyR

140 organization did not result from channel degradation, but rather indicate progressive dispersion of RyR

141 clusters into more numerous, smaller groupings.

We next performed 3D correlative imaging of RyRs and the t-tubular network to distinguish between dyadic and non-dyadic ("orphaned") RyRs (Fig. 1f). Based on these analyses, the majority of RyR clusters were classified as dyadic, and this proportion was observed to be similar under control and isoproterenol-treatment conditions ( $83 \pm 1.6 \%$ and $84 \pm 1.0 \%$, respectively; Fig. 1g). Importantly, RyR cluster dispersion was found to be restricted to RyRs within dyads (control: $9.0 \pm 0.5$ vs $60 \mathrm{~min}$ isoproterenol: $6.7 \pm 0.4 \mathrm{RyRs} / \mathrm{cluster}$, Fig. $1 \mathrm{~h}$ ). It should be noted that the above analyses were performed on RyR clusters within the cell interior. Interestingly, the organization of surface RyR clusters associated with the cardiomyocyte sarcolemma was unaffected by $\beta$-AR activation (Supplementary Fig. 1), suggesting that RyR dispersion is a phenomenon restricted to internal, dyadic RyRs. We thus focused only on interior RyR clusters for the remainder of the study. 

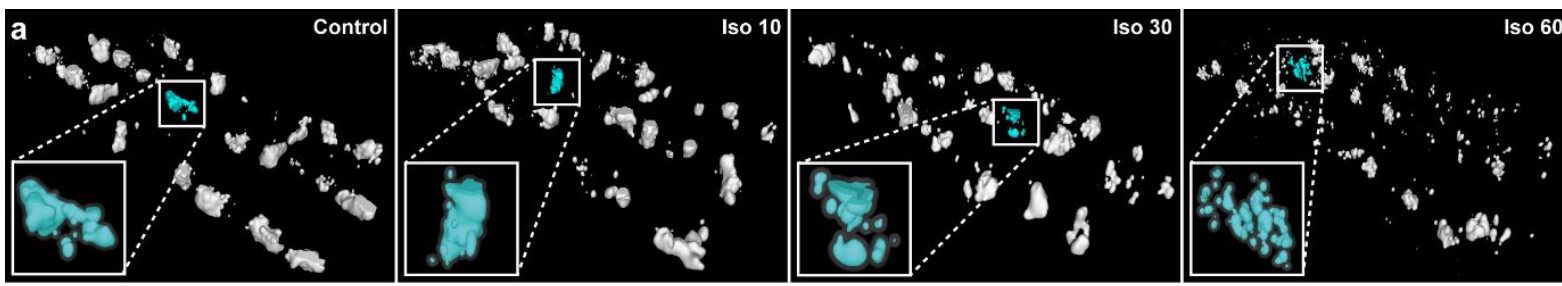

b

c

d
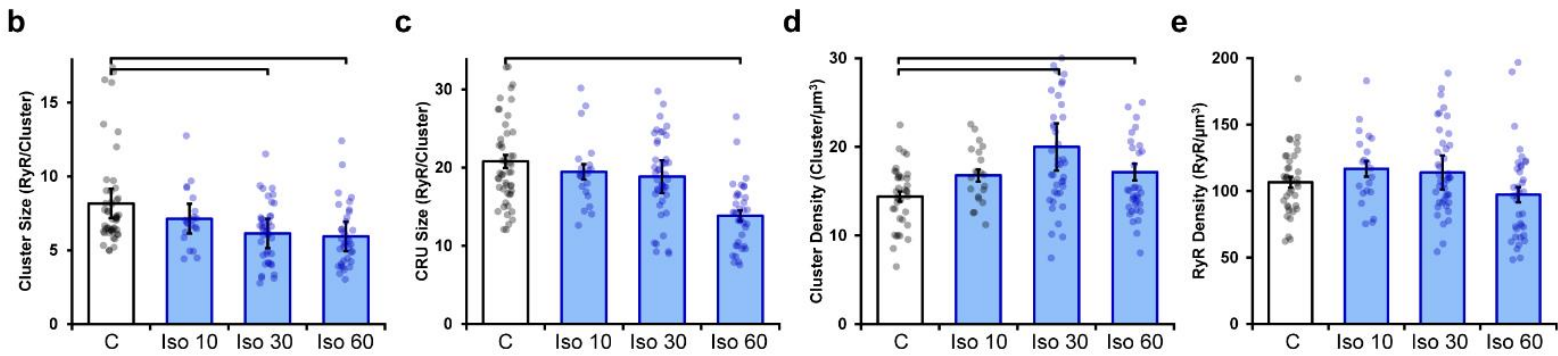

f
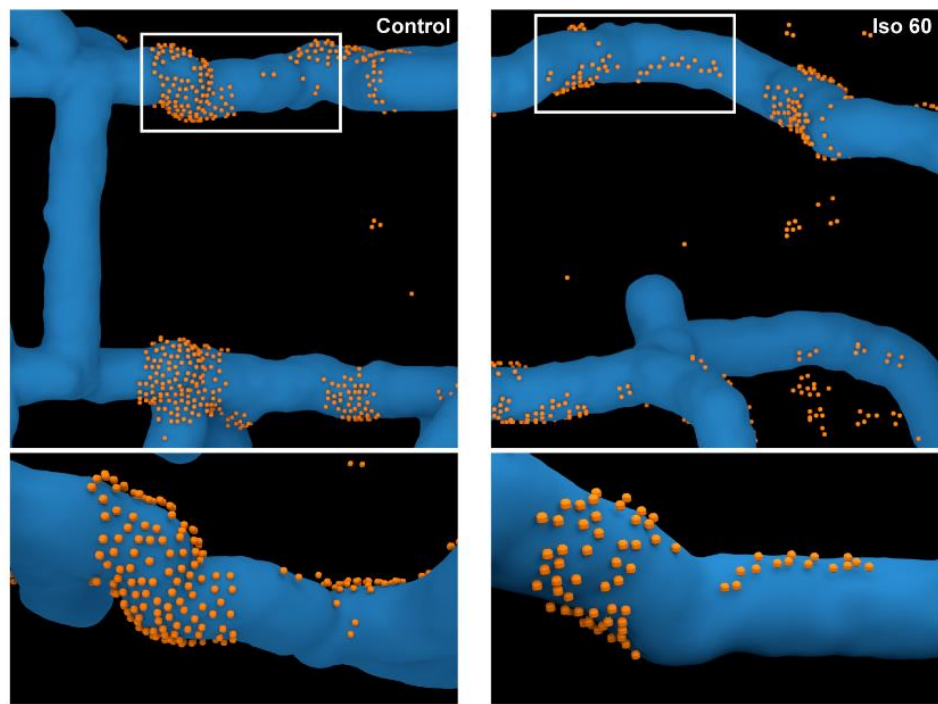

g
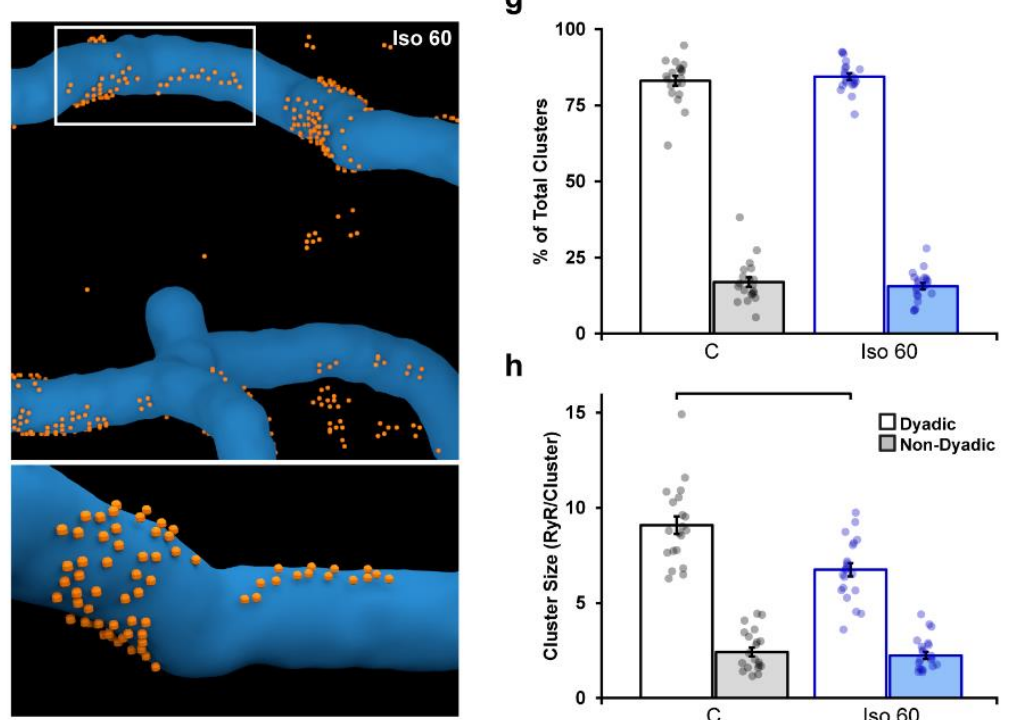

h

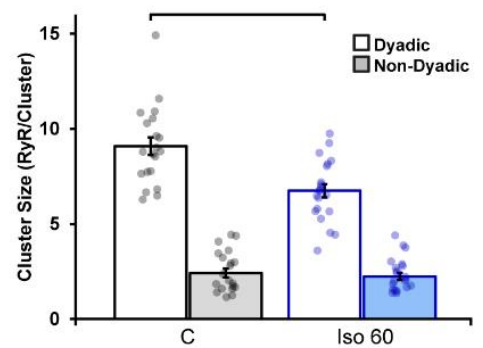

Figure 1: Prolonged $\beta$-adrenergic receptor $(\boldsymbol{\beta}-\mathbf{A R})$ activation disperses RyR clusters. (a) Representative reconstructions of internal RyR clusters based on 3D dSTORM in isolated rat cardiomyocytes. Control conditions are compared with isoproterenol treatment $(100 \mathrm{nM})$ of varying duration (10, 30 and 60 minutes). The insets depict single CRUs, which encompass RyR clusters with edge-to-edge distances $\leq 100 \mathrm{~nm}$. (b-d) Quantification of the dSTORM data revealed progressive dispersion of RyR clusters during isoproterenol treatment, as indicated by measurements of RyR cluster size, CRU size, and cluster density. (e) RyR density was unchanged. (Control: $\mathrm{n}_{\text {cells }}=50, \mathrm{n}_{\text {hearts }}=6$; Iso 10: $\mathrm{n}_{\text {cells }}=21, \mathrm{n}_{\text {hearts }}=3$; Iso 30: $\mathrm{n}_{\text {cells }}=$ 43, $\mathrm{n}_{\text {hearts }}=4$; Iso 60: $\mathrm{n}_{\text {cells }}=37, \mathrm{n}_{\text {hearts }}=5$ ). (f) Correlative imaging of the transverse tubular network (confocal microscopy) and RyRs (dSTORM) was employed to create 3D reconstructions of dyadic and non-dyadic CRUs (T-tubules: blue; RyRs: orange). (g) Similar proportions of clusters were characterized as dyadic under control and following 60 min isoproterenol. (h) Cluster size measurements indicated that dispersion during isoproterenol occurred exclusively within dyads (Control: $\mathrm{n}_{\text {cells }}$ $=20, \mathrm{n}_{\text {hearts }}=3$; ISO 60: $\mathrm{n}_{\text {cells }}=21, \mathrm{n}_{\text {hearts }}=3$ ). 
bioRxiv preprint doi: https://doi.org/10.1101/2022.02.18.481024; this version posted February 19, 2022. The copyright holder for this preprint (which was not certified by peer review) is the author/funder, who has granted bioRxiv a license to display the preprint in perpetuity. It is made available under aCC-BY 4.0 International license.

\section{$167 \mathrm{Ca}^{2+}$ /calmodulin-dependent protein kinase II (CaMKII) activation mediates isoproterenol-induced RyR cluster dispersion}

169 In cardiomyocytes, it is well established that a substantial portion of $\beta$-AR signaling is mediated via $\mathrm{CaMKII}^{18}$, and includes CaMKII-mediated phosphorylation of RyRs ${ }^{19,20}$. Indeed, using flow cytometry analysis, we observed increased RyR phosphorylation at the CaMKII site ser-2814, that was particularly prominent during early stages of isoproterenol treatment (Supplementary Fig. 2a-c). We therefore hypothesized that isoproterenol-induced cluster dispersion is, at least in part, driven by the actions of CaMKII. To this end, we first tested effects of the CaMKII competitive inhibitor autocamtide-2-related inhibitory peptide (AIP, $2 \mu \mathrm{M}$ ) following an initial hour-long isoproterenol incubation (i.e., $1 \mathrm{~h}$ isoproterenol followed by $1 \mathrm{~h}$ isoproterenol + AIP). We indeed found that the addition of AIP markedly reversed cluster dispersion (Fig. 2a). This was reflected by a significant increase in both RyR cluster size (Fig. 2d) and CRU size (Fig. 2e), as well as a significant reduction in RyR cluster density (Fig. 2f). Furthermore, direct activation of CaMKII through the Epac2 pathway using 8-CPT-cAMP $(10 \mu \mathrm{M})^{21}$ consistently reproduced the RyR cluster dispersion observed during isoproterenol stimulation (Fig. 2b, mean measurements in Fig. 2d-f). RyR dispersion was again reversed by the addition of AIP posttreatment in these cells. To further corroborate our results, we indirectly stimulated CaMKII activity by applying a low concentration of caffeine $(0.5 \mathrm{mM})$ to cardiomyocytes to increase spontaneous $\mathrm{Ca}^{2+}$ release from the $\mathrm{SR}^{22}$. We found that caffeine application gave rise to significant reductions in both RyR cluster and CRU size, as well as a significant increase in cluster density (Fig. 2c, mean data in Fig. 2d-f). Again, these changes were reversed when AIP was administered.

While these findings suggest that isoproterenol-induced cluster dispersion is dependent on CaMKII, it remained unclear whether these effects can be directly attributed to its phosphorylation of RyRs. To address this issue, we examined RyR cluster characteristics in cardiomyocytes isolated from transgenic mice in which the CaMKII phosphorylation site on RyR is either constitutively active (S2814D) or genetically ablated (S2814A). We found that the RyR arrangement in the phosphomimetic S2814D mutant cells at baseline resembled the fragmented RyR organization observed in wild type (WT) animals after isoproterenol treatment (Fig. 2g, h). Indeed, cluster size (Fig. 2j), CRU size (Fig. 2k), and cluster density (Fig. 21) were all similar in WT + ISO and S2814D cardiomyocytes, and significantly different from untreated WT cells. Furthermore, application of isoproterenol to S2814D cardiomyocytes did not lead to significant additional RyR dispersion, and only tendencies toward smaller cluster and CRU sizes (Fig. 2h, j-1). In contrast, cardiomyocytes from non-phosphorylatable S2814A mutant mice exhibited similar RyR organization as in WT mice at baseline (Fig. 2i). Administering isoproterenol to this group promoted a significant reduction in cluster size (Fig. 2j) and increased cluster density (Fig. 21). These findings mirror observations in rat cardiomyocytes, where CaMKII inhibition did not fully reverse RyR dispersion in several experiments (Fig. 2d-f). Taken together, these results support that CaMKII-dependent RyR phosphorylation during prolonged $\beta$-AR stimulation is a key driver for cluster dispersion, but that there are likely additional contributing mechanisms. 

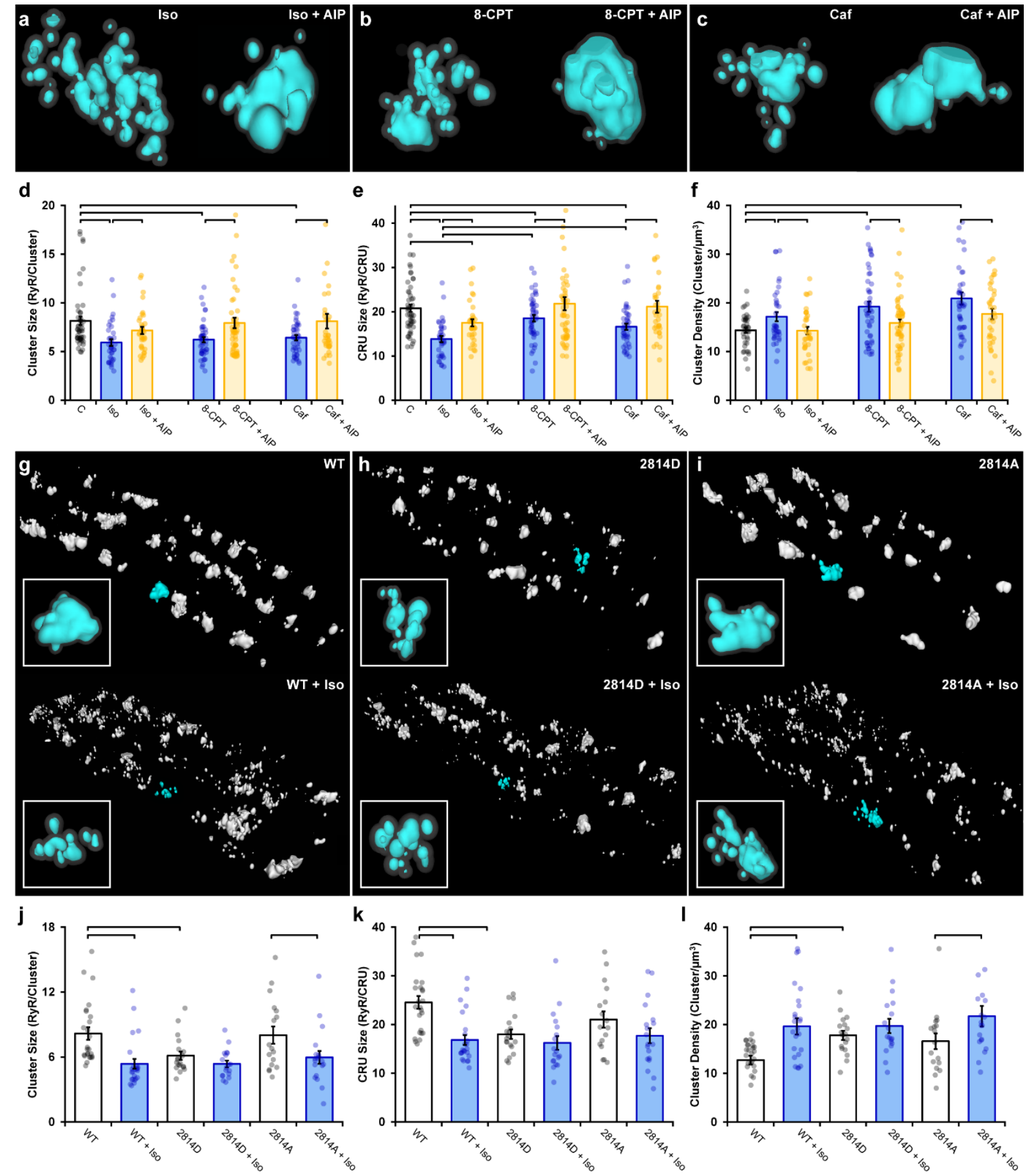

Figure 2: $\mathrm{Ca}^{2+} /$ calmodulin-dependent protein kinase II (CaMKII) activation promotes RyR cluster dispersion. (a-c) Representative CRUs imaged in cardiomyocytes treated with isoproterenol $(100 \mathrm{nM}), 8$-CPT $(10 \mu \mathrm{M})$, or caffeine $(0.5 \mathrm{mM})$ for 1 hour (left panels) or with with inclusion of the CaMKII inhibitor AIP $(2 \mu \mathrm{M})$ for an additional hour (right panels). (d-f) Induced RyR cluster dispersion was reversed by CaMKII inhibition, as indicated by measurements of RyR cluster size, CRU size, and cluster density. $\left(\right.$ Control: $\mathrm{n}_{\text {cells }}=50, \mathrm{n}_{\text {hearts }}=6$; Iso: $\mathrm{n}_{\text {cells }}=37, \mathrm{n}_{\text {hearts }}=5$; Iso + AIP: $\mathrm{n}_{\text {cells }}=37$, $\mathrm{n}_{\text {hearts }}=5$; 8-CPT: $\mathrm{n}_{\text {cells }}=48, \mathrm{n}_{\text {hearts }}=5 ;$ 8-CPT + AIP: $\mathrm{n}_{\text {cells }}=52, \mathrm{n}_{\text {hearts }}=5 ;$ Caffeine: $\mathrm{n}_{\text {cells }}=42, \mathrm{n}_{\text {hearts }}=4 ;$ Caffeine + AIP: $\mathrm{n}_{\text {cells }}=35, \mathrm{n}_{\text {hearts }}=$ 3). (g-h) Representative images of RyR organization in cardiomyocytes from wild type mice (WT) and transgenic mice constitutively activated (S2814D) or genetically ablated (S2814A) phosphorylation at S2814. Images and mean data (j-I) are presented under baseline conditions and following $60 \mathrm{~min}$ isoproterenol stimulation. $\left(\mathbf{W T}: \mathrm{n}_{\text {cells }}=25, \mathrm{n}_{\text {hearts }}=2\right.$; WT + Iso: $\mathrm{n}_{\text {cells }}=24, \mathrm{n}_{\text {hearts }}=2 ;$ S2814D: $\mathrm{n}_{\text {cells }}=19, \mathrm{n}_{\text {hearts }}=2 ; \mathbf{S 2 8 1 4 D}+$ Iso: $\mathrm{n}_{\text {cells }}=18, \mathrm{n}_{\text {hearts }}=2 ; \mathbf{S 2 8 1 4 A}: \mathrm{n}_{\text {cells }}=17, \mathrm{n}_{\text {hearts }}=2 ; \mathbf{S 2 8 1 4 A}$ 
bioRxiv preprint doi: https://doi.org/10.1101/2022.02.18.481024; this version posted February 19,2022 . The copyright holder for this preprint (which was not certified by peer review) is the author/funder, who has granted bioRxiv a license to display the preprint in perpetuity. It is made available under aCC-BY 4.0 International license.

\section{Protein Kinase A (PKA)-dependent phosphorylation also contributes to RyR cluster dispersion during $\beta$-AR activation}

Following $\beta$-AR stimulation, increased cyclic AMP levels lead to PKA-dependent phosphorylation of the RyR at ser-2808 ${ }^{17}$ (Supplementary Fig. 2d). Based on our above results, we hypothesized that in parallel to CaMKII-mediated RyR phosphorylation, activation of PKA following isoproterenol application also contributes to RyR cluster dispersion. Indeed, $\beta$-AR-induced cluster dispersion was reversed by the addition of the PKA inhibitor H89 (Fig. 3a), as reflected by significantly increased cluster size (Fig. 3c) and CRU size (Fig. 3d), and reduced cluster density (Fig. 3e). To further validate an involvement of PKA, we quantified RyR arrangement following application of the selective PKA activator 6MB-cAMP (6MB, $100 \mu \mathrm{M})^{23,24}$. As illustrated in Fig. 3b, 6MB-cAMP treatment prompted RyR dispersion comparable to that elicited by isoproterenol. These changes were again reversible by co-incubating 6MB with $\mathrm{H} 89$ following an initial treatment period with 6MB alone (Fig. 3c-e). As in experiments investigating effects of CaMKII inhibition (Fig. 2), it should be noted that PKA inhibition did not fully restore the RyR configuration at the CRU level. These data support that CaMKII- and PKA-dependent phosphorylation of RyRs concertedly drive RyR dispersion during prolonged $\beta$-AR stimulation.

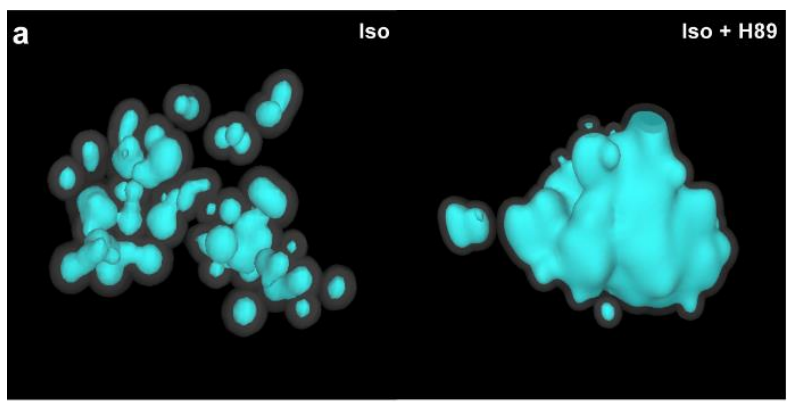

C

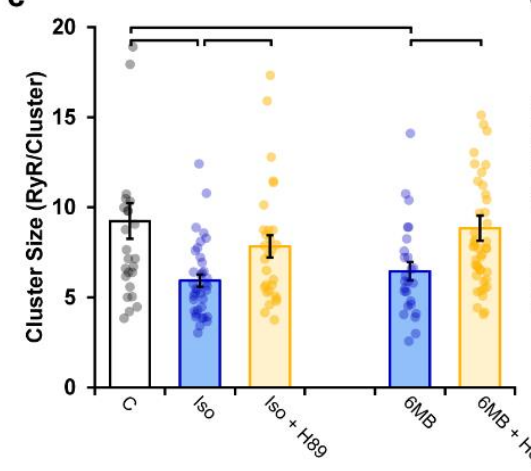

d

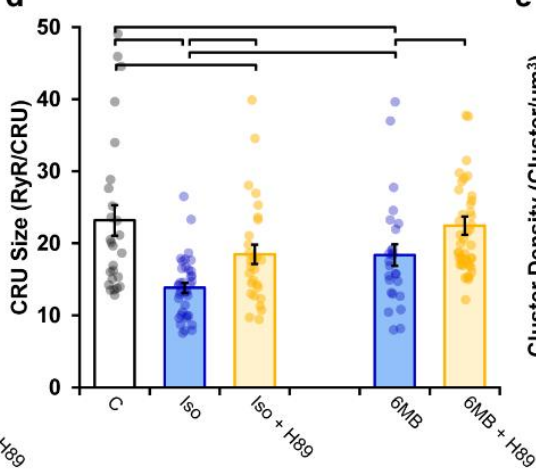

e
$6 \mathrm{MB}$
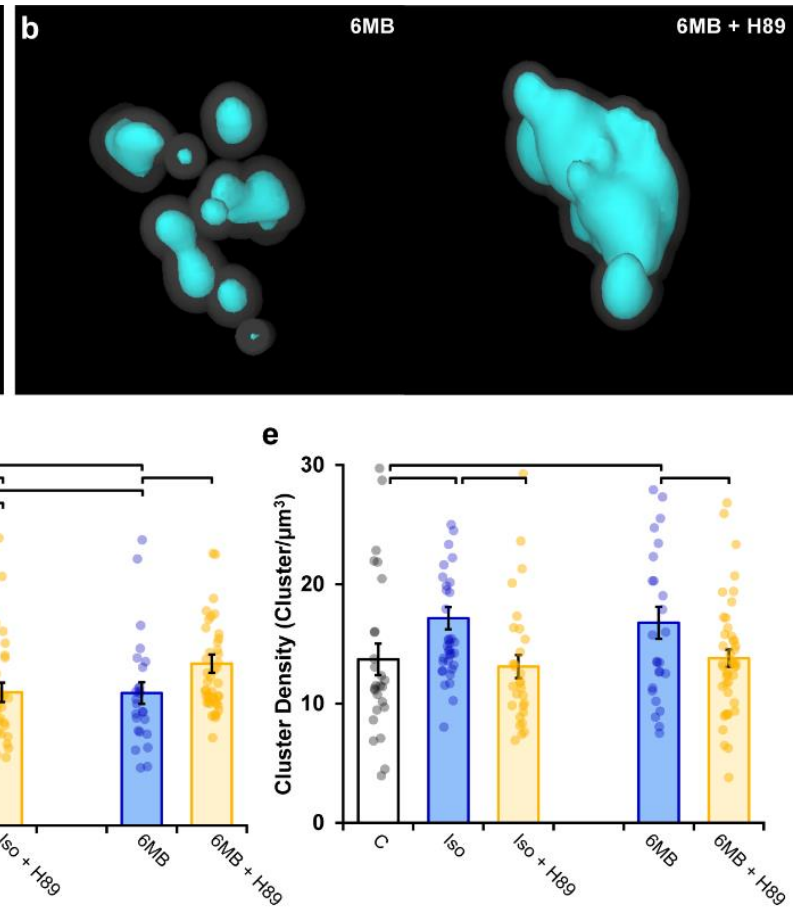

Figure 3: Protein kinase A (PKA)-dependent phosphorylation also promotes RyR cluster fragmentation during $\beta$-AR activation. (a) Representative CRUs indicate that PKA inhibition (H89, $10 \mu \mathrm{M})$ reverses isoproterenol-induced cluster dispersion. (b) Direct activation of PKA using 6MB-cAMP $(100 \mu \mathrm{M})$ can also induce cluster dispersion, and is similarly reversed when followed by H89 application. (c-e) Mean data comparing cluster size, CRU size and cluster density between groups (Control: $\mathrm{n}_{\text {cells }}=26, \mathrm{n}_{\text {hearts }}=3$; Iso: $\mathrm{n}_{\text {cells }}=37$, $\mathrm{n}_{\text {hearts }}=5 ;$ Iso + H89: $\mathrm{n}_{\text {cells }}=30, \mathrm{n}_{\text {hearts }}=4 ; 6 \mathrm{MB}: \mathrm{n}_{\text {cells }}=26, \mathrm{n}_{\text {hearts }}=3$; $6 \mathrm{MB}+$ H89: $\left.\mathrm{n}_{\text {cells }}=44, \mathrm{n}_{\text {hearts }}=4\right)$. 


\section{$246 \beta$-stimulation-induced RyR dispersion alters $\mathbf{C a}^{2+}$ sparks, transients, and waves}

247 We next assessed the functional consequences of RyR dispersion. We examined spontaneous $\mathrm{Ca}^{2+}$ 248 sparks since these events occur almost exclusively at dyads ${ }^{25}$, where RyR arrangements appear to be 249 sensitive to $\beta$-AR stimulation (Fig. 1f). Previous work has shown that acute $\beta$-AR activation increases 250 the frequency of spontaneous $\mathrm{Ca}^{2+}$ sparks, while diverse effects on spark geometry are reported ${ }^{26-28}$. We 251 hypothesized, however, that RyR dispersion during prolonged $\beta$-AR stimulation would slow spark 252 kinetics and increase $\mathrm{Ca}^{2+}$ leak, as released $\mathrm{Ca}^{2+}$ propagates between multiple clusters. Representative $253 \mathrm{Ca}^{2+}$ spark recordings from control and isoproterenol-treated cells are presented in Fig. 4a and b, 254 respectively, with corresponding spark time courses presented in the right panels. We observed that while spark frequency was augmented throughout the isoproterenol treatment period (Fig. 4c), there was indeed a progressive slowing of both spark rise time (Fig. 4d) and duration (Fig. 4e). Increased overall spark geometry (Fig. 4f, g) during prolonged isoproterenol treatment was linked to progressively augmented $\mathrm{Ca}^{2+}$ leak, as calculated based on spark mass and frequency (Fig. 4h). Importantly, reversal of RyR dispersion by co-incubation of cells with either AIP or H89 after the initial isoproterenol treatment also reversed slowing of spark kinetics and isoproterenol-induced $\mathrm{Ca}^{2+}$ leak (Fig. 4d-h).

Changes in RyR localization and function during prolonged $\beta$-AR stimulation were additionally linked to alterations in cell-wide $\mathrm{Ca}^{2+}$ transients (Fig. 4i). As expected, isoproterenol treatment acutely increased $\mathrm{Ca}^{2+}$ transient magnitude, however this reversed with continued exposure (Fig. 4j). This pattern was mirrored by changes in SR $\mathrm{Ca}^{2+}$ content (Supplementary Fig. 3), which was also initially increased by heightened SERCA activity during isoproterenol exposure, but then began to decline as RyR $\mathrm{Ca}^{2+}$ leak progressively increased. Similar changes were observed in the synchrony of $\mathrm{Ca}^{2+}$ release across the cell. $\mathrm{Ca}^{2+}$ release tended to be more synchronous at early stages of isoproterenol treatment (Fig. 41), in agreement with previous work indicating that increasing $\mathrm{SR} \mathrm{Ca}^{2+}$ content and $\mathrm{RyR}$ sensitization enable more homogeneous $\mathrm{Ca}^{2+}$ transients ${ }^{22,29}$. However, after prolonged $\beta$-AR stimulation, RyR dispersal, slowed $\mathrm{Ca}^{2+}$ spark kinetics, and declining SR content were coupled to a reversal of the synchronization of $\mathrm{Ca}^{2+}$ release observed at earlier time points (Fig. 4l), and a modest slowing of the rising phase of the transient (Fig. 4k).

The above data are consistent with the notion that RyR dispersion gradually counters the stimulatory effects of $\beta$-AR stimulation on CICR, perhaps in a protective manner. To further examine this hypothesis, we investigated the propensity for spontaneous $\mathrm{Ca}^{2+}$ waves during isoproterenol treatment (Fig. 4i). Indeed, while we observed an expected initial increase in $\mathrm{Ca}^{2+}$ wave incidence following isoproterenol application, this reversed after 60 min as wave frequency was reduced by $\sim 30 \%$ (Fig. $4 \mathrm{~m}$ ). Thus, sufficient dispersion of RyRs and accompanying weakening of CICR inhibits wave propagation, 
bioRxiv preprint doi: https://doi org/10.1101/2022.02.18.481024: this version posted February 19, 2022. The copyright holder for this

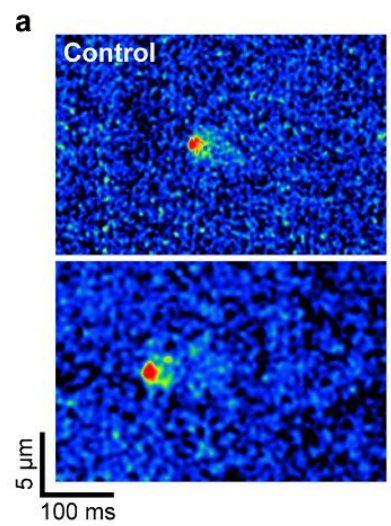

c

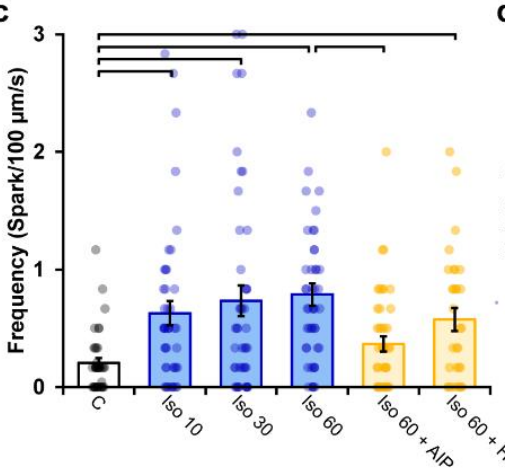

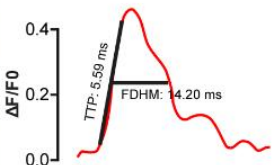

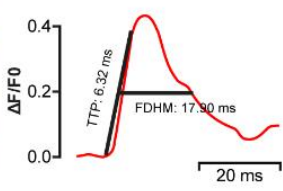

d

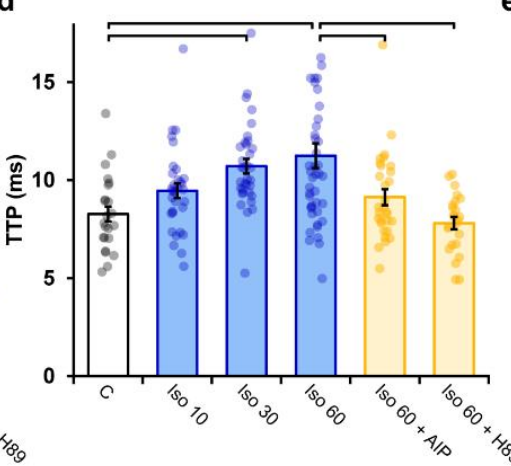

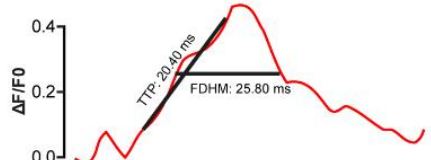
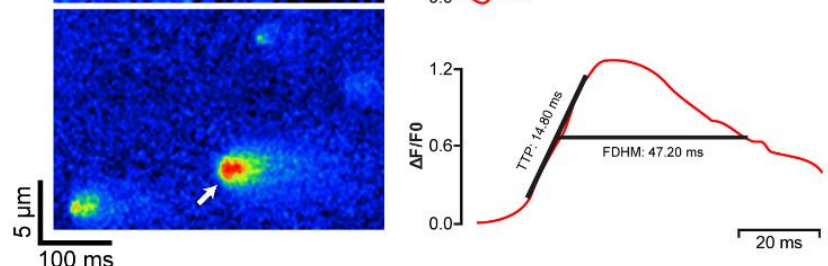

e

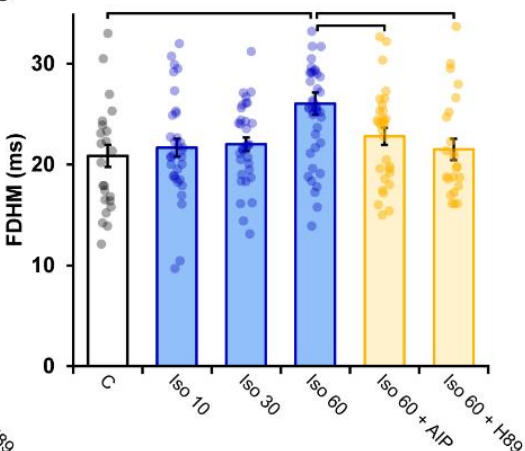

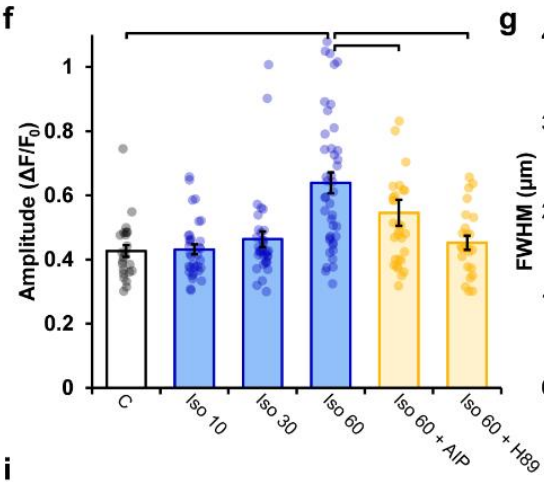

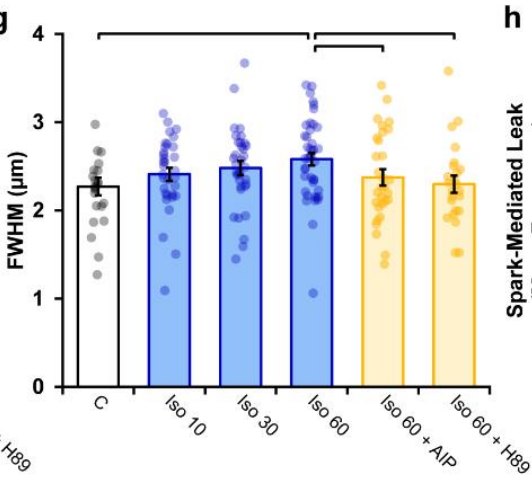

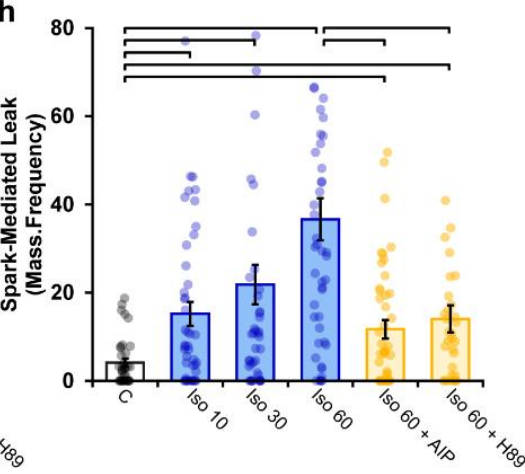
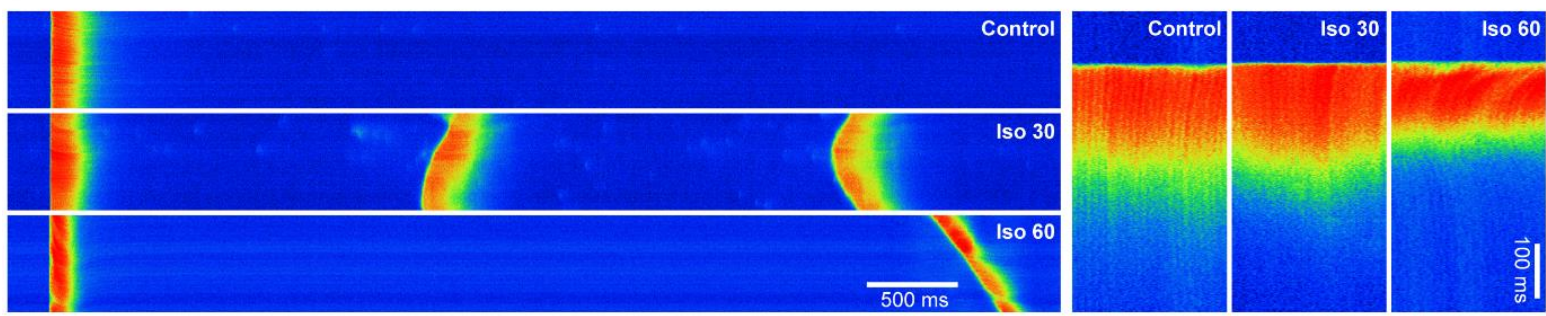

j

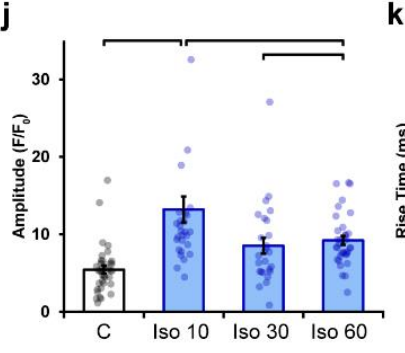

k

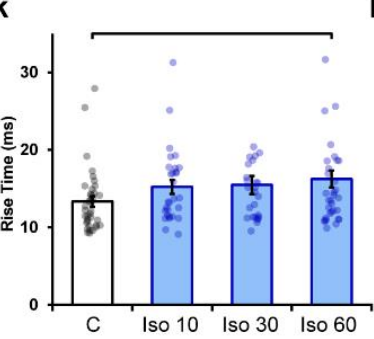

I

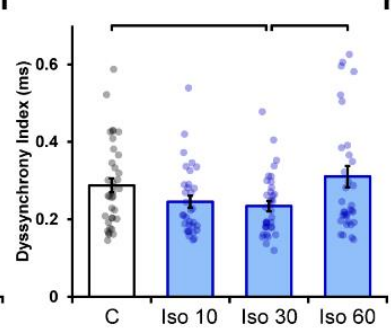

m

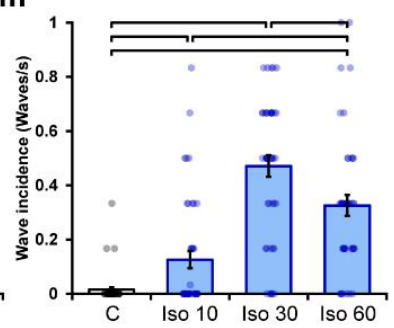

Figure 4: Effects of RyR dispersion of intracellular $\mathrm{Ca}^{2+}$ homeostasis are reversed by CaMKII or PKA inhibition. (a, b) Representative examples of $\mathrm{Ca}^{2+}$ sparks of control and isoproterenol (60 min)-treated cardiomyocytes obtained by confocal line-scan imaging. The time course for each spark is shown at right, with indicated TTP and FDHM measurements. (c-h) Mean data indicate that increasing spark frequency, geometry, and slowing of kinetics during isoproterenol were all reversed by 
$\mathrm{n}_{\text {cells }}=47, \mathrm{n}_{\text {hearts }}=3$; Iso $60+$ AIP: $\mathrm{n}_{\text {cells }}=45, \mathrm{n}_{\text {hearts }}=3$; Iso $60+\mathrm{H} 89 \mathrm{n}_{\text {cells }}=31, \mathrm{n}_{\text {hearts }}=2$ ). (i) Representative linescan images illustrating the last in a series of electrically-paced $\mathrm{Ca}^{2+}$ transients, followed by a pause to examine $\mathrm{Ca}^{2+}$ wave generation. Enlargements of the $\mathrm{Ca}^{2+}$ transients are shown at right to highlight differences in $\mathrm{Ca}^{2+}$ release synchrony. (j-l) Mean data showed that initial increases in $\mathrm{Ca}^{2+}$ transient amplitude and synchrony were reversed with continued exposure to isoproterenol, while overall release kinetics slowed. (Control: $\mathrm{n}_{\text {cells }}=38, \mathrm{n}_{\text {hearts }}=3$; Iso 10: $\mathrm{n}_{\text {cells }}=35, \mathrm{n}_{\text {hearts }}=3$; Iso 30: $\mathrm{n}_{\text {cells }}=$ 36 , $\mathrm{n}_{\text {hearts }}=3$; Iso $60: \mathrm{n}_{\text {cells }}=41, \mathrm{n}_{\text {hearts }}=3$ ). $(\mathbf{m}) \mathrm{Ca}^{2+}$ wave incidence increased during early time points following isoproterenol treatment, but then reversed. (Control: $\mathrm{n}_{\text {cells }}=43$, nhearts $=3$; Iso 10: $\mathrm{n}_{\text {cells }}=44$, $\mathrm{n}_{\text {hearts }}=3$; Iso 30: $\mathrm{n}_{\text {cells }}=46$, nhearts $=3$; Iso 60: $\mathrm{n}_{\text {cells }}=44, \mathrm{n}_{\text {hearts }}=3$ ).

\section{RyR cluster dispersion lowers CRU excitability in a mathematical model}

To support the above correlative data linking RyR arrangement and $\mathrm{Ca}^{2+}$ homeostasis, we employed a mathematical reaction-diffusion model of $\mathrm{Ca}^{2+}$ release in the CRU. Using paired images of RyRs (3D dSTORM) and t-tubules (confocal microscopy), we generated four computational CRU geometries that were representative of the range of RyR dispersion observed in cells (Fig. 5a, Supplementary Table 1). For each geometry, a series of 400 stochastic spark simulations was performed with and without the regulatory effects of $\beta$-AR stimulation on RyR sensitivity and SR load included (Fig. 5b). Other model parameters are described in Supplementary Tables 2 and 3.

Each simulated $\mathrm{Ca}^{2+}$ release event was classified as being an observable spark event $\left(\Delta \mathrm{F} / \mathrm{F}_{0} \geq 0.3\right)$, a sub-spark event $\left(\mathrm{Ca}^{2+}\right.$ quark $\left.{ }^{30}\right)$, or a failed spark for which negligible $\mathrm{Ca}^{2+}$ release occurred $\left(\Delta \mathrm{F} / \mathrm{F}_{0} \leq\right.$ 0.1 ; Fig. 5b). The proportion of observable sparks elicited was used as an estimate of spark fidelity (Fig. 5e). With baseline model parameters, RyR cluster dispersion was observed to have a large impact on CRU excitability. Indeed, spark fidelity declined steadily with increased fragmentation (95\% CI in solid CRU: $28.6-37.8 \%$ vs majorly fragmented CRU: 0-1.2\%, Fig. 5e). However, $\beta$-AR stimulation augments both RyR sensitivity and, particularly at early stages, $\mathrm{SR} \mathrm{Ca}^{2+}$ content. Increasing channel sensitivity and $\mathrm{Ca}^{2+}$ release flux accordingly in the model improved inter-RyR communication, and increased spark fidelity. In the cases of the minorly- and moderately-dispersed CRUs, these effects more than compensated for the effects of RyR fragmentation, as spark fidelity even exceeded values obtained in solid CRUs at baseline (Fig. 5b, e). However, CRUs with greater degrees of RyR dispersion continued to exhibit lower fidelity of spark generation than those with more compact arrangements.

Analyzing only successful spark events revealed that RyR cluster dispersion also influenced spark amplitude (Fig. 5c) and kinetics (Fig. 5d). Individual simulation results are presented alongside experimental data in Supplementary Fig. 4. While minor to moderate degrees of RyR dispersion had rather little impact on spark amplitude, majorly fragmented CRUs exhibited markedly smaller events (solid w/ $\beta$-AR: $\Delta \mathrm{F} / \mathrm{F}_{0}=0.52 \pm 0.001$, majorly fragmented $\mathrm{w} / \beta-\mathrm{AR}: \Delta \mathrm{F} / \mathrm{F}_{0}=0.37 \pm 0.004$, Fig. $5 \mathrm{c}$ ). This decrease was attributed to a shift from an all-or-none pattern of RyR activation in solid CRU arrangements to partial activation in fragmented units (Fig. 5b). The time to peak (TTP) of sparks increased with the degree of dispersion in our model (Fig. 5d) and, under simulated $\beta$-AR activation, this was primarily due to the sensitized RyRs' improved ability to sustain regenerative release between clusters. Indeed, in simulations where SR load was systematically altered with constant $\mathrm{RyR} \mathrm{Ca} \mathrm{Ca}^{2+}$ sensitivity, spark kinetics were not markedly affected (Supplementary Fig. 5). These findings support that RyR cluster dispersion during prolonged $\beta$-AR activation is directly linked with slower, and lower magnitude $\mathrm{Ca}^{2+}$ sparks observed experimentally. proportion of total RyR-mediated $\mathrm{Ca}^{2+}$ leak that is "silent", rather than spark-mediated (Fig. 5f). Due to declining spark fidelity, we observed that although total $\mathrm{Ca}^{2+}$ released from a given CRU decreased with greater fragmentation, the proportion of leak which is silent increased dramatically. When 
338 including the effects of $\beta$-AR stimulation, fidelity increased and the ratio of silent leak was strongly 339 reduced, becoming close to zero for the mildly to moderately fragmented CRUs. For the majorly 340 fragmented CRU, however, there remained a considerable amount of silent leak (Majorly fragmented 341 w/B-AR: $37.5 \%$ silent leak, Fig. 5f) linked to partial activation of the $\mathrm{CRU}$ ( $\mathrm{Ca}^{2+}$ quarks).

343 Taken together, the modeling results indicate that $\mathrm{RyR} \mathrm{Ca}^{2+}$ leak shifts from spark-mediated to silent 344 leak when CRUs are dispersed, reducing the fidelity of spark generation. Thus, while RyR 345 phosphorylation during acute $\beta$-AR stimulation markedly enhances spark fidelity, gradual CRU 346 fragmentation during continued stimulation reverses these changes. An accompanying reduction in SR 347 content is expected to exacerbate declining spark fidelity during prolonged $\beta$-AR stimulation. 348 Furthermore, when sparks are successfully elicited within dispersed CRU geometries, $\mathrm{Ca}^{2+}$ release is 349 slowed and of lower magnitude. 
a
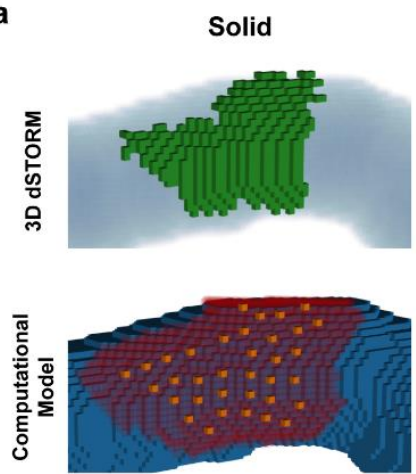

Thresholded dSTORM
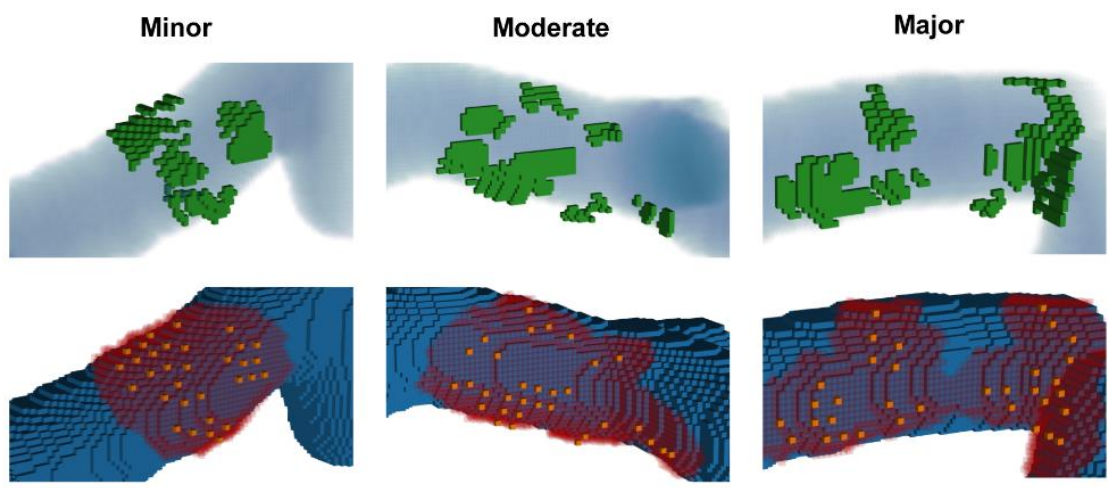

Reconstructed t-tubule structure

RyR channel pores

Cleft space
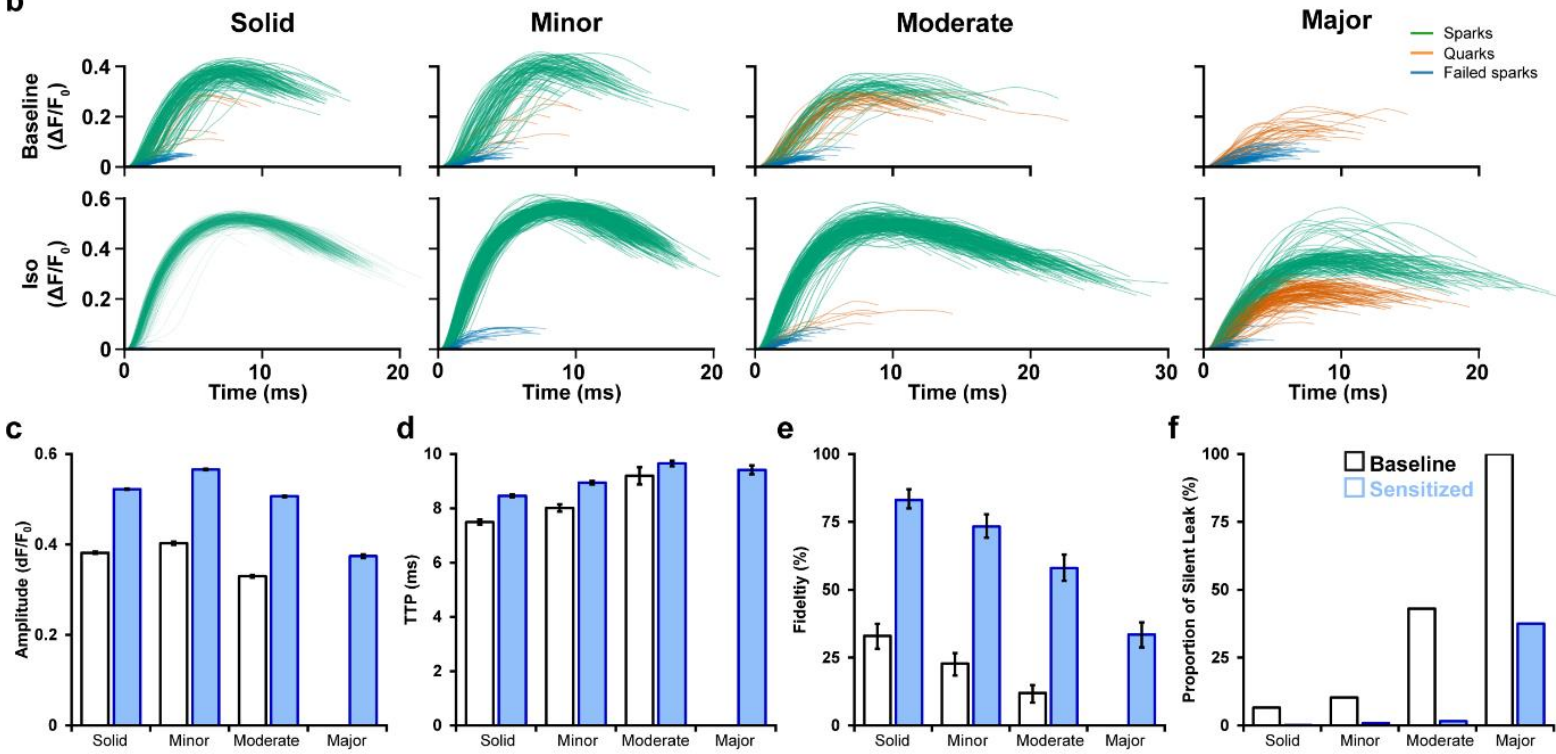

Figure 5. Mathematical modeling linking changes in RyR configuration and function during $\beta$-adrenergic stimulation. (a) 3D computational volumetric meshes built from correlative imaging of the t-tubular network (confocal microscopy) and RyRs (3D dSTORM). Constructed geometries consisted of 10x10x10 nm voxels and a 1x1x1 $\mu \mathrm{m}$ volume. The four geometries were chosen to represent the range of cluster dispersion seen in imaging, see Table 1 for details. (Top row) Reconstructed ttubular structures (blue) are illustrated with superimposed thresholded RyR signals (green). (Bottom Row) Corresponding computational geometries, with indicated RyR channel pores (orange) and cleft space (red) defined by morphological dilation around the RyRs. The four geometries were selected to represent the range of RyR dispersion observed in experiments, ranging from solid to majorly-fragmented CRUs (see methods). (b) 400 stochastic spark simulations were performed in each geometry with baseline model parameters, and another 400 were conducted with the regulatory effects of $\beta$-AR stimulation added (RyR sensitization and increased SR content). The $\Delta \mathrm{F} / \mathrm{F}_{0}$ time course is shown for each spark. The maximal amplitude used to define the release as an experimentally observable spark-event $\left(\Delta \mathrm{F} / \mathrm{F}_{0} \geq 0.3\right)$, a sub-spark quark event $\left(\Delta \mathrm{F} / \mathrm{F}_{0} \geq 0.1\right)$, or a failed spark $\left(\Delta \mathrm{F} / \mathrm{F}_{0}<0.1\right)$. (c, $\left.\mathbf{d}\right)$ Mean amplitude and time to peak (TTP) measurements for observable sparks. (e, f) The classification of release into spark and non-spark events was used to estimate spark fidelity and the ratio of leak that is "silent" (i.e., sub-spark release) in each simulation case. Error bars in C and D show standard error, while the bars in E indicate the 95\% Agresti-Coull confidence interval.

\section{CaMKII and PKA activation promote RyR dispersion and $\mathrm{Ca}^{2+}$ dysregulation during} heart failure (HF)

We next examined whether dispersion of RyRs reported during $\mathrm{HF}^{11}$ similarly results from RyR hyperphosphorylation. Previous work has indeed linked CaMKII ${ }^{15,16}$ and PKA activity ${ }^{17}$ to RyR dysfunction in this disease. We first examined RyR phosphorylation status in rats with post-infarction HF compared to sham-operated controls (see Supplementary Table 4 for animal characteristics). Western blotting revealed no significant change in total RyR expression, and only a tendency toward increased PKA 
bioRxiv preprint doi: https://doi.org/10.1101/2022.02.18.481024; this version posted February 19,2022 . The copyright holder for this preprint (which was not certified by peer review) is the author/funder, who has granted bioRxiv a license to display the preprint in perpetuity. It is made available under aCC-BY 4.0 International license.

375 phosphorylation at S2808 (Fig. 6a, b - source data 1). However, phosphorylation at S2814 - a site linked to CaMKII activation - was significantly increased by $\sim 55 \%$ in HF (Fig. $6 \mathrm{~b}-$ source data 1 ).

3D dSTORM imaging of Sham and HF cardiomyocytes (Fig. 6c) revealed significant RyR dispersion in failing cells, manifested at both the cluster level (Fig. 6d; Sham: $7.93 \pm 0.46$ vs HF: $5.16 \pm 0.27$ RyRs/Cluster) and CRU level (Fig. 6e; Sham: $20.79 \pm 1.09$ vs HF: $14.82 \pm 1.01$ RyRs/CRU). These structural changes are consistent with a previous 2D super-resolution study which utilised the same pathological model ${ }^{11}$. Next, we subjected cells to either CaMKII or PKA inhibition to assess whether disrupted RyR organisation could be reversed. Indeed, inhibiting CaMKII phosphorylation with AIP in HF cells restored RyR organization to Sham values, as evidenced by a $49 \%$ increase in cluster size and a $47 \%$ increase in CRU size (Fig. 6d, e). To a lesser extent, the addition of $\mathrm{H} 89$ to failing cells also significantly reversed RyR dispersion at the cluster level (28\% increase) and CRU level (19\% increase). Correlative imaging of RyRs and T-tubules (Fig. 6c) revealed a significantly smaller proportion of dyadic clusters in HF compared to Sham (Fig. 6f); an expected finding since reduced t-tubule density in this model results in the formation of "orphaned" RyRs ${ }^{31,32}$. The smaller RyR clusters observed in failing cells were found to exclusively occur at dyadic sites (Sham: $9.09 \pm 0.47$ vs HF: $6.81 \pm 0.44$ RyRs/cluster), where dispersed RyR arrangements were again reversible by CaMKII or PKA inhibition (Fig. 6g). The striking similarity of these findings to experiments employing prolonged isoproterenol exposure (Figs. 1-4) supports that fragmentation of RyR clusters during HF results from prolonged $\beta$ AR activation stimulation in this condition, linked to the activation of both CaMKII and PKA.

Finally, we examined whether alterations in $\mathrm{RyR} \mathrm{Ca}^{2+}$ release during HF could be linked to RyR phosphorylation and dispersion. Representative recordings of $\mathrm{Ca}^{2+}$ sparks are presented in Fig. 6h, together with temporal profiles shown at right. Failing cardiomyocytes exhibited significantly increased frequency (Fig. 6i), slower rise time (Fig. 6j), and longer duration sparks (Fig. 6k) than in sham cells, which summated to marked augmentation of spark-mediated leak (Fig. 61). All of these changes were reversed by inhibiting CaMKII or PKA, although the effects of CaMKII inhibition were the most marked (Fig. 6h-1). Overall $\mathrm{Ca}^{2+}$ transients were of lower magnitude in failing cardiomyocytes (Fig. 6m), and $\mathrm{Ca}^{2+}$ release was observed to be slowed and dyssynchronous (Fig. 6n, 6o, see Supplementary Fig. 6 for representative examples). CaMKII inhibition again reversed changes in $\mathrm{Ca}^{2+}$ transient kinetics and synchrony in failing cells to values similar to Sham (Fig. 6n, o). Taken together, these findings identify phosphorylation-dependent RyR dispersion as a key driver of RyR dysfunction in HF, and particularly implicate CaMKII overactivity in this process. 
bioRxiv preprint doi: https://doi org/10.1101/2022.02.18.481024: this version posted February 19, 2022. The copyright holder for this preprint (which was not certified by peer review) is the author/funder, who has granted bioRxiv a license to display the preprint in perpetuity. It is made available under aCC-BY 4.0 International license.
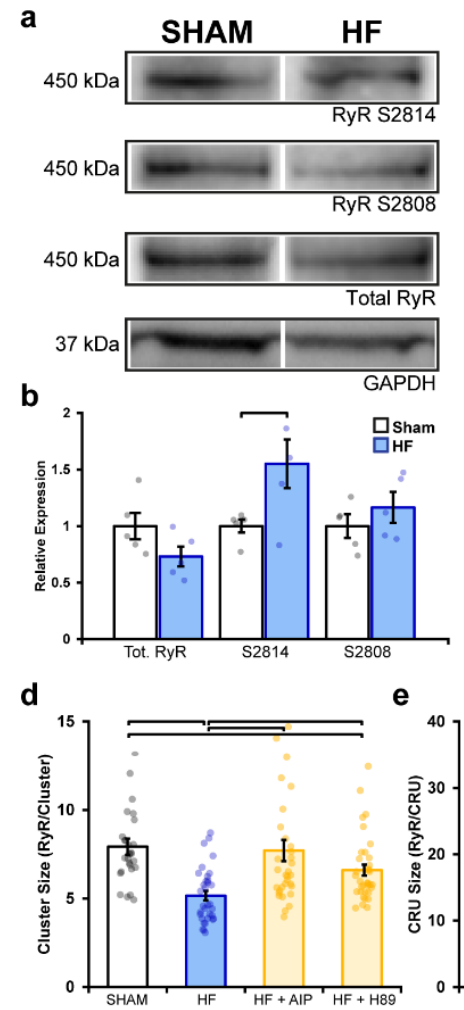

e
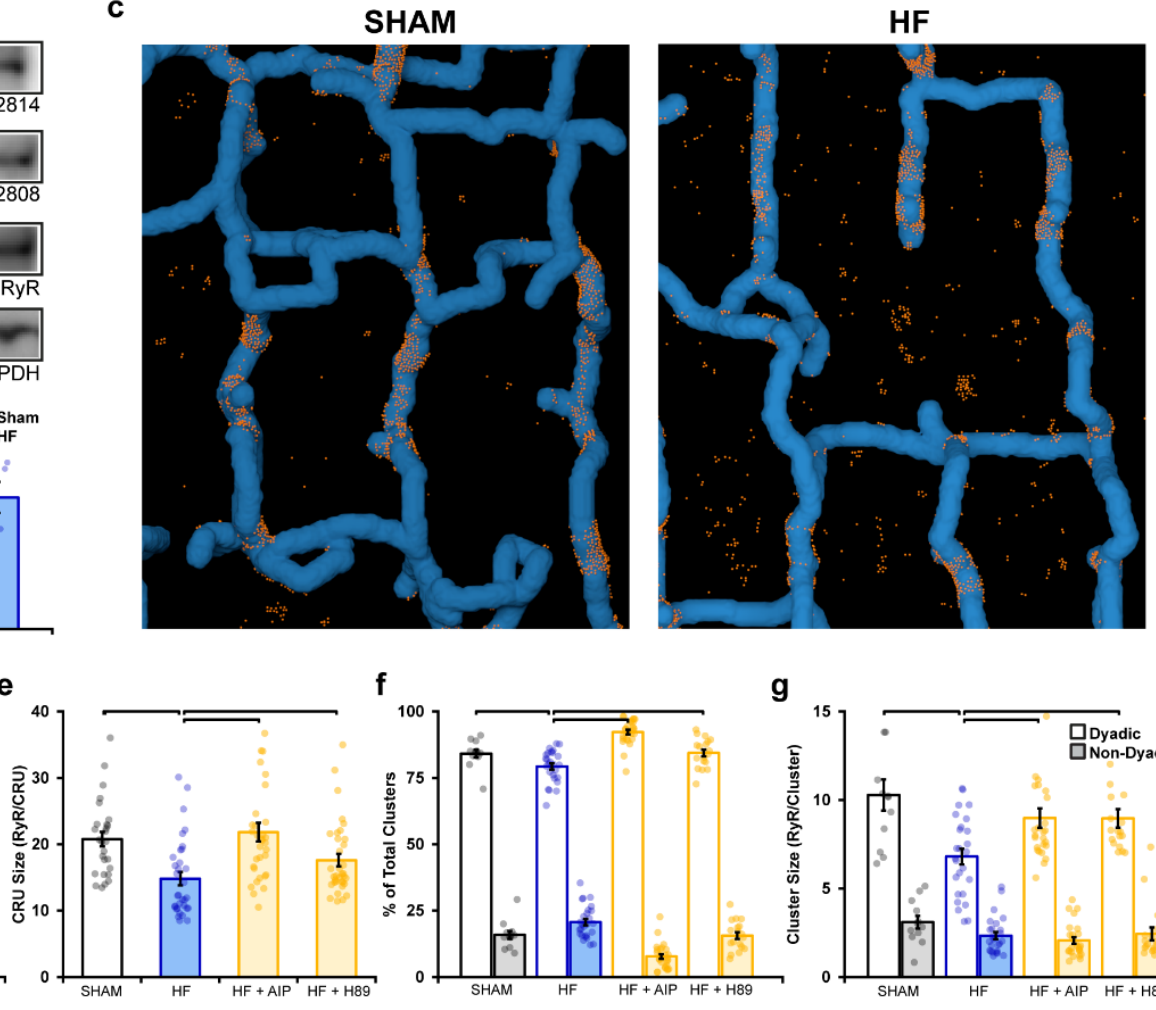

g

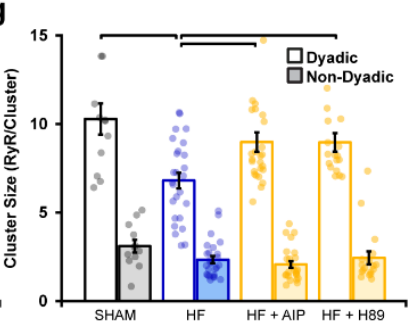

h
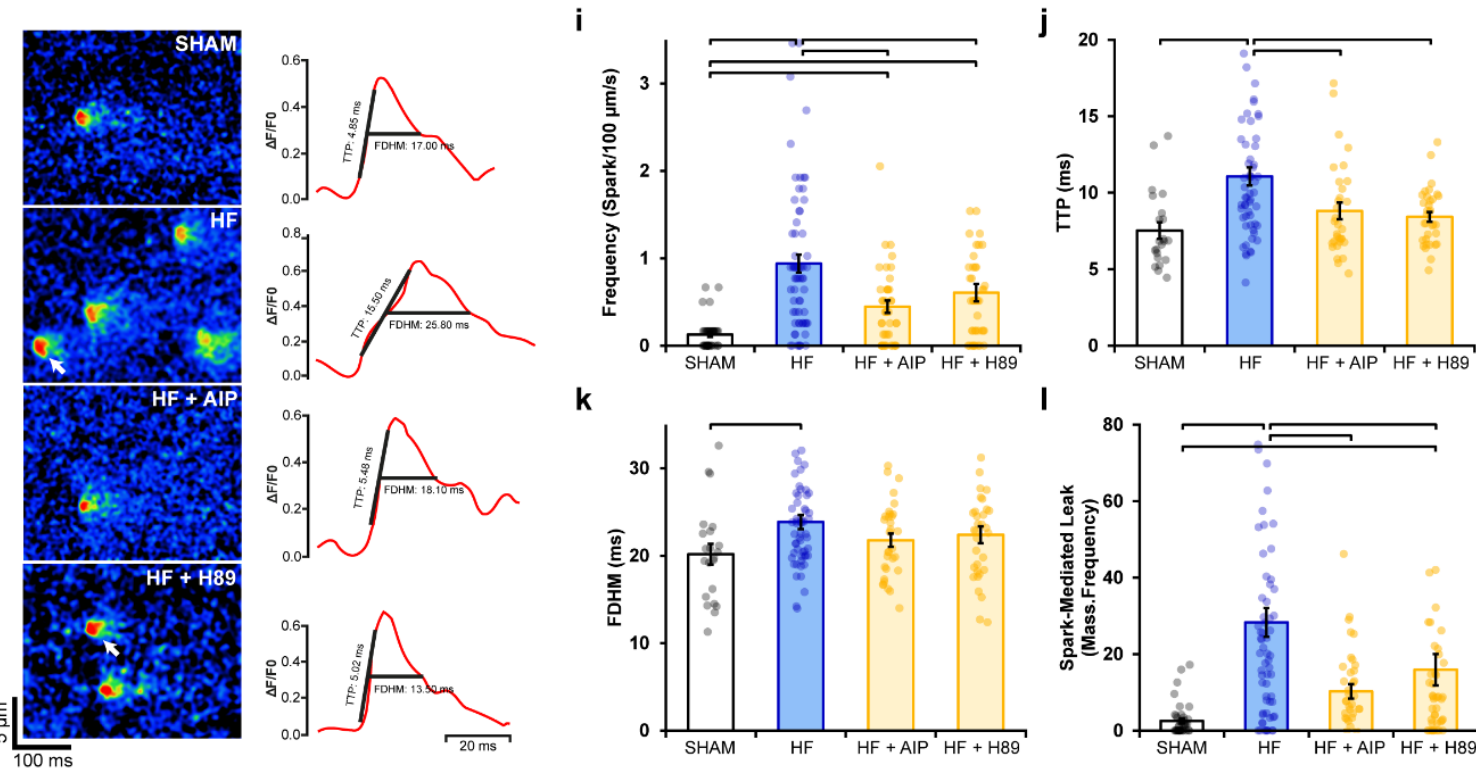

k
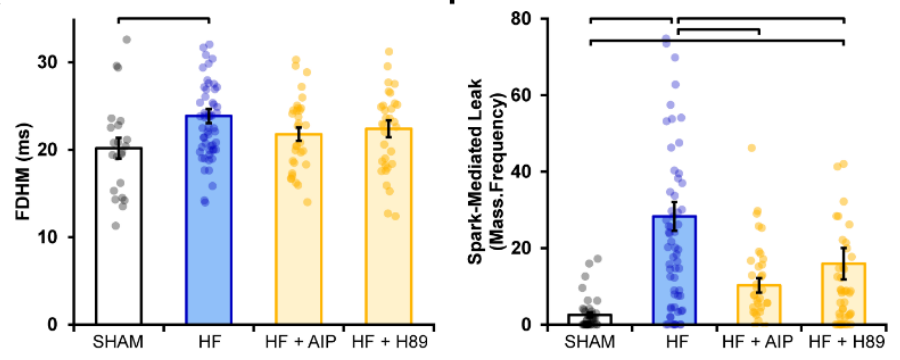

m
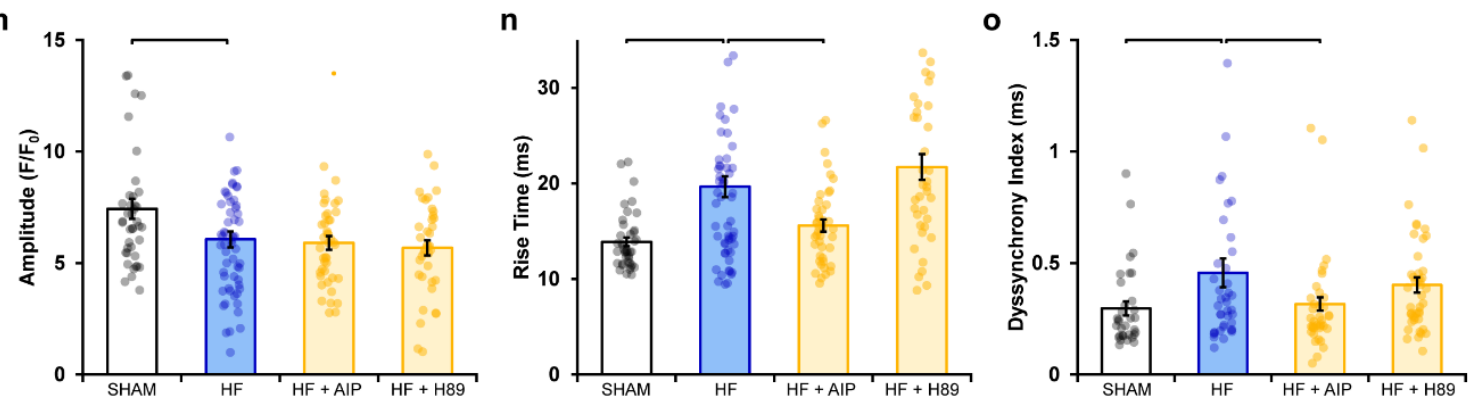

Figure 6. Remodeling of RyR organization and function during heart failure (HF) are reversed by inhibiting phosphorylation. (a, b) Representative Western blots and mean data comparing the relative expression of CaMKIIphosphorylated RyR (S2814), PKA-phosphorylated RyR (S2808) and total RyR in Sham and HF cardiomyocytes. (Sham: $n_{\text {hearts }}=5$; HF: nhearts $=5$ ). (c) 3D reconstruction of dyads in Sham and failing cells based on correlative imaging of t-tubules 
density in cardiomyocytes from Sham and HF cells, at baseline and following inhibition of CaMKII (AIP) or PKA (H89). (Sham: $\mathrm{n}_{\text {cells }}=27, \mathrm{n}_{\text {hearts }}=4 ; \mathrm{HF}: \mathrm{n}_{\text {cells }}=33, \mathrm{n}_{\text {hearts }}=4 ; \mathrm{HF}+$ AIP: $\left.\mathrm{n}_{\text {cells }}=33, \mathrm{n}_{\text {hearts }}=4 ; \mathrm{HF}+\mathrm{H} 89: \mathrm{n}_{\text {cells }}=35, \mathrm{n}_{\text {hearts }}=4\right) .(\mathbf{h})$ Representative $\mathrm{Ca}^{2+}$ spark examples from Sham and HF cardiomyocytes with or without CaMKII inhibition (AIP) or PKA inhibition (H89). The time course of each spark is shown at right. (i-l) Mean data comparing spark duration, time to peak, frequency and spark-mediated leak in Sham and HF cells, at baseline and following treatment with AIP or PKA (Sham: $\mathrm{n}_{\text {cells }}$ $\left.=42, \mathrm{n}_{\text {hearts }}=3 ; \mathrm{HF}: \mathrm{n}_{\text {cells }}=65, \mathrm{n}_{\text {hearts }}=5 ; \mathrm{HF}+\mathrm{AIP}: \mathrm{n}_{\text {cells }}=63, \mathrm{n}_{\text {hearts }}=5 ; \mathrm{HF}+\mathrm{H} 89: \mathrm{n}_{\text {cells }}=43, \mathrm{n}_{\text {hearts }}=3\right)$. (m-o) Mean measurements of cell-wide $\mathrm{Ca}^{2+}$ transient magnitude, TTP (rise time), and dyssynchrony in Sham and HF cells, in the presence and absence of AIP or PKA (Sham: $\mathrm{n}_{\text {cells }}=41, \mathrm{n}_{\text {hearts }}=4 ; \mathrm{HF}: \mathrm{n}_{\text {cells }}=59, \mathrm{n}_{\text {hearts }}=4$; HF + AIP: $\mathrm{n}_{\text {cells }}=43, \mathrm{n}_{\text {hearts }}=4 ; \mathrm{HF}+\mathrm{H} 89$ : $\mathrm{n}_{\text {cells }}=38, \mathrm{n}_{\text {hearts }}=3$ ).

\section{Discussion}

In this work, we identify an important new mechanism by which RyR organization and function are controlled by $\beta$-AR stimulation. Specifically, we show that during prolonged $\beta$-AR activation, as occurs during HF, there is a progressive dispersion of RyR clusters. This fragmentation is driven by both CaMKII- and PKA-dependent phosphorylation of the channel, and is reversible by inhibiting these kinases. Functionally, our experimental and modeling results indicate that dispersed CRUs exhibit increased "silent" $\mathrm{Ca}^{2+}$ leak, and low spark fidelity. When $\mathrm{Ca}^{2+}$ sparks are successfully generated by dispersed CRUs, they exhibit slowed kinetics and reduced magnitude. These changes are in turn coupled with smaller and desynchronized $\mathrm{Ca}^{2+}$ transients, which are hallmarks of failing cells. However, we also observed protective actions of CRU dispersion, as lowered spark fidelity and magnitude protected against $\mathrm{Ca}^{2+}$ wave generation. Thus, RyR dispersal appears to be aimed at curbing arrhythmogenesis during prolonged $\beta$-AR stimulation, but comes at the expense of systolic function, and this is particularly detrimental in the setting of HF.

While the precise nature of inter-RyR dynamics remains incompletely understood, several previous studies have suggested that there are important structural determinants of CRU function ${ }^{11,33-35}$. Of note, recent work by Galice et al. (2018) measured RyR cluster size and $\mathrm{Ca}^{2+}$ sparks simultaneously, finding that spark frequency depends steeply on cluster size, while spark amplitudes appear to be independent of size above a certain threshold. The authors argued that RyR cluster sizes must strike a balance between being sufficiently large to ensure high-fidelity CICR, but not so large as to promote arrhythmogenic behavior. When RyR channels are sensitized or $\mathrm{Ca}^{2+}$ levels are elevated, this balance shifts, increasing the propensity for sparks, diastolic leak and waves. Extending from these findings, our present experimental and mathematical modeling results suggest that RyR cluster dispersion compensates for this shift by physically inhibiting $\mathrm{Ca}^{2+}$ wave propagation, but to the detriment of spark fidelity.

Our data show that marked RyR cluster dispersion occurs only after prolonged $\beta$-AR stimulation, which likely explains why previous, acute-treatment studies have not detected similar changes in RyR arrangement or function. For example, Asghari et al. ${ }^{14}$ recently performed detailed analysis of RyR positioning, but within only 10 minutes of channel phosphorylation. Nevertheless, these authors did observe changes in the precise positioning of neighboring RyRs within this timeframe, as a checkerboard arrangement of RyRs was favored over side-by-side arrangements. Both arrangements involve SRPY and P1 domain-domain interactions between adjacent receptors ${ }^{36}$. A shift in the configuration of these domains, for example during phosphorylation, might be envisioned to reduce the stability of RyR dimerization, i.e., reducing RyR "stickiness". Indeed, Asghari et al ${ }^{14}$ observed a modest rightward shift in the RyR-RyR nearest neighbor distance early after initiation of phosphorylation, which could represent the earliest stages of cluster breakup that we have observed at later time points. However, neither we (Supplementary Fig. 1) nor Asghari et al observed similar rearrangement of RyRs at the cell surface during $\beta$-AR stimulation, suggesting that there are unique features of internal RyRs 
and/or their partner proteins that render their positioning phosphorylation-sensitive. FKBP12.6 is one such candidate, as it has been shown to be directly involved in domain-domain interactions between adjacent $\mathrm{RyRs}^{36}$, and to fine-tune RyR positions in directions which are opposite those of phosphorylation ${ }^{14}$.

It is well established that prolonged $\beta$-AR stimulation is linked to time-dependent desensitization of the signaling pathway; a process that involves $G$ protein-coupled receptor kinases and $\beta$-arrestins which promote internalization and downregulation of the receptors ${ }^{37}$. Our results indicate that this process was likely initiated in our experiments, since RyR phosphorylation at ser-2814 and ser-2808 both exhibited bell-shaped responses during the $1 \mathrm{~h}$ treatment protocol (Supplementary Fig. 2). A similar response was observed in measurements of SERCA activity (Supplementary Fig. 3c). Importantly, RyR phosphorylation by CaMKII and/or PKA remained elevated above control levels at the end of the protocol. These heightened phosphorylation levels apparently remain sufficient to support RyR dispersion, since inhibition of either kinase following $\beta$-AR stimulation caused RyRs to re-cluster (Fig. 2a-f, 3). The regulatory role of CaMKII and PKA on RyR dispersion was further corroborated by experiments showing that selective activation of either kinase induced fragmented CRU arrangements. Furthermore, transgenic mice with constitutively active S2814 were found to have significantly dispersed clusters at baseline compared to their wild-type counterparts (Fig. 2g-1). Notably, genetic ablation of S2814 did not appear to inhibit the effects of $\beta$-AR stimulation, which continued to reduce RyR cluster size and increase cluster density. Taken together, these findings point to a more complicated mechanism responsible for cluster dispersion than direct phosphorylation of the RyR at the S2814 and S2808 residues by CaMKII and PKA, respectively. Rather, it seems likely that CaMKII and PKA act interdependently on the RyR. Other studies have also suggested cross-talk between the two sites, with one observing changes to S2814 phosphorylation in a S2808 mutant ${ }^{38}$, and another demonstrating increased PKA activity at S2808 following S2814 phosphorylation ${ }^{39}$. In HF cells, we detected an increase in RyR phosphorylation at S2814, but only a minor trend towards higher phosphorylation at S2808. Still, inhibition of either CaMKII or PKA reversed cluster dispersion in these cells, although the extent of reversion following AIP administration was more complete than that obtained with H89. These results appear to be consistent with studies suggesting that PKA may not be the principal determinant of RyR hyper-phosphorylation in $\mathrm{HF}^{16,40}$, but also indicate that CaMKII and PKA control RyR clustering in a coordinated manner.

Although we observed a close relationship between RyR organization and function in our experiments, we relied on mathematical modeling to directly interrogate the functional implications of cluster dispersion vs other regulatory effects of $\beta$-AR stimulation. The modeling results indicated that RyR cluster fragmentation is sufficient to significantly weaken intra-CRU RyR communication. Notably, these structural-driven changes in function were opposite those induced by regulatory changes, as increased RyR sensitivity to cytosolic $\mathrm{Ca}^{2+}$ and increased SR $\mathrm{Ca}^{2+}$ content increased intra-CRU RyR communication. These observations are consistent with the view that while acute $\beta$-AR stimulation effectively augments CICR, progressive RyR dispersion and accompanying decline of SR $\mathrm{Ca}^{2+}$ content during continued exposure serves to gradually reverse these changes, as $\mathrm{Ca}^{2+}$ release fidelity, magnitude, and kinetics are compromised (Fig. 5c-e). In healthy cardiomyocytes, this functional decline was not overly severe, as $\mathrm{Ca}^{2+}$ transients measured experimentally remained somewhat larger than in untreated cells, and $\mathrm{Ca}^{2+}$ release kinetics were only modestly slowed (Fig. 4j, k). However, during HF, more detrimental consequences of RyR dispersion are expected, as SR content is reduced below normal levels. Thus, compensatory changes in RyR function observed in healthy cells during $\beta$-AR stimulation are expected to be less present, and impairment of CICR more robust. 
511 Experimental studies have frequently linked increases in spark frequency to higher incidence of $\mathrm{Ca}^{2+}$ 512 waves ${ }^{41,42}$. Here, we also report a marked increase in both spark and wave incidence during the early

513 stages of $\beta$-AR stimulation (Fig. 4i, m). However, while both spark frequency and mass continued to

514 increase with prolonged treatment, wave incidence significantly declined. While this finding may at

515 first seem counterintuitive, a similar 'paradoxical' observation was reported in a canine model of left

516 ventricular hypertrophy ${ }^{43}$. The authors hypothesized that disconnects between junctional SR clusters

517 and LTCCs could lead to spatial heterogeneity in SR $\mathrm{Ca}^{2+}$ content, enabling regions with lower SR $\mathrm{Ca}^{2+}$

518 to act as "fire breaks" against wave propagation. We suggest that an analogous mechanism explains our

519 findings, but which may include both reduced SR content and dispersed CRUs acting as a physical

520 barrier to $\mathrm{Ca}^{2+}$ wave propagation. Since accumulating evidence supports that RyR positioning is tightly

521 regulated, we believe that RyR dispersion in healthy cardiomyocytes is, by design, aimed at curbing wave generation during prolonged $\beta$-AR stimulation. This is an important consideration if we are to consider targeting RyR localization in disease. While increasing RyR-RyR "stickiness" in failing cells would be expected to augment RyR clusters sizes, inter-channel collaboration, and systolic function, our data suggest that such effects may also increase susceptibility to arrhythmia.

In summary, we observed that RyR clusters progressively disperse during protracted $\beta$-AR activation, due to channel phosphorylation by both CaMKII and PKA. Experimental and mathematical modeling results linked this dyadic rearrangement to declining efficacy of CICR, including slowed and reduced magnitude $\mathrm{Ca}^{2+}$ release, but also protection against pro-arrhythmic $\mathrm{Ca}^{2+}$ waves. These findings have important implications for $\mathrm{HF}$, and other conditions such as catecholaminergic polymorphic ventricular tachycardia, which are associated with increased phosphorylation of RyRs and risk of arrhythmia.

\section{Material and Methods}

\section{Ethical approval}

All animal experiments were performed in accordance with the Norwegian Animal Welfare Act and NIH Guidelines, and were approved by the Ethics Committee of the University of Oslo and the Norwegian animal welfare committee (FOTS ID 20208). The majority of the experiments were performed on adult male Wistar rats (250-350 g) purchased from Janvier Labs (Le Genest-Saint-Isle, France). Rats were group housed at $22^{\circ} \mathrm{C}$ on a $12 \mathrm{~h}: 12 \mathrm{~h} \mathrm{light-dark} \mathrm{cycle,} \mathrm{with} \mathrm{free} \mathrm{access} \mathrm{to} \mathrm{food} \mathrm{and}$ water. Cardiomyocytes isolated from transgenic RyR2-S2814D and RyR2-2814A mice ${ }^{44}$ were provided by the laboratory of Xander Wehren (Baylor College of Medicine, Texas, United States). A total of 64 rats and 6 mice were used in this study. The authors understand the ethical principles under which Nature Medicine operates and declare that our work complies with this animal ethics checklist.

\section{Rat model of post-myocardial infarction congestive HF}

Left coronary artery ligation was performed to induce large anterolateral myocardial infarctions in male Wistar rats ${ }^{45}$. Development of HF was verified six weeks later using a Vevo 2100 echocardiography imaging system (VisualSonics, Toronto, Canada). HF animals were selected based on established criteria $^{46}$, including dilation of the left atrium (diameter $>5 \mathrm{~mm}$ ) and infarction size above $40 \%$ (Supplementary Table 4). Sham-operated rats served as controls. Sample sizes were determined by power analysis, assuming that only $50 \%$ of post-infarction animals would be included in the final data set, and based on a pilot project of variability in CRU morphology in healthy controls.

\section{Rat Ventricular Cardiomyocyte Isolation}


bioRxiv preprint doi: https://doi.org/10.1101/2022.02.18.481024; this version posted February 19, 2022. The copyright holder for this preprint (which was not certified by peer review) is the author/funder, who has granted bioRxiv a license to display the preprint in perpetuity. It is made available under aCC-BY 4.0 International license.

free oxygenated solution (in mmol/L: $140 \mathrm{NaCl}, 5.4 \mathrm{KCl}, 0.5 \mathrm{MgCl}_{2}, 0.4 \mathrm{NaH}_{2} \mathrm{PO}_{4}, 5$ HEPES, 5.5 glucose $\mathrm{pH}$ 7.4). Once the heart had been cleared of blood, perfusion was switched to the same solution containing collagenase type II ( $1.8 \mathrm{mg} / \mathrm{ml}$, Worthington Biochemical Corporation) for 10-12 mins at 37 ${ }^{\circ} \mathrm{C}$. Following digestion, left ventricular tissue was dissected and finely cut into $3-4 \mathrm{~mm}^{3}$ pieces. A secondary digestion was performed to liberate additional cells from tissue by transferring approximately $8 \mathrm{ml}$ of tissue and collagenase solution to a $10 \mathrm{ml}$ Falcon tube containing $0.2 \mathrm{mg}$ DNase (LS002006, Worthington) in $500 \mu \mathrm{l}$ BSA. Cells were subsequently filtered and allowed to pellet in $0.2 \mathrm{mmol} / \mathrm{L} \mathrm{Ca}^{2+}$.

\section{Immunofluorescence Labeling}

Isolated cardiomyocytes were washed with Dulbecco's PBS (No. 4387, Biowhittaker), fixed with 4\% PFA for $10 \mathrm{~min}$, quenched with $100 \mu \mathrm{mol} / \mathrm{L}$ glycine for $10 \mathrm{~min}$, and permeabilised with $1 \%$ triton X100 for $10 \mathrm{~min}$. The cells were then plated on glass bottom dishes (No 1.5, Ø $14 \mathrm{~mm}, \gamma$-irradiated, Martek Corporation) that had been coated with laminin (mouse, BD Biosciences) and blocked using ImageiT ${ }^{\text {TM }}$ FX Signal Enhancer (Thermo Fisher Scientific) prior to immunolabeling.

For RyR visualisation, cells were incubated with mouse anti-RyR (1:100, MA3-916, Thermo Fisher Scientific) overnight at $4{ }^{\circ} \mathrm{C}$. For t-tubule imaging, cells were incubated overnight at $4{ }^{\circ} \mathrm{C}$ in a mixture of rabbit anti-Cav-3 (1:100, ab2912, Abcam) antibody and a custom rabbit anti-NCX1 antibody as previously described ${ }^{5}(1: 100$, Genscript Corporation, Piscataway, NJ). Secondary antibody labeling was carried out using donkey anti-mouse Alexa Fluor 647 (1:200, A-21237, Thermo Fisher Scientific) and goat anti-rabbit Alexa Fluor 488 (1:200, A-11070, Thermo Fisher Scientific) antibodies for $1 \mathrm{~h}$ at room temperature. Both primary and secondary antibodies were diluted in a blocking buffer consisting of $2 \%$ goat serum and $0.02 \% \mathrm{NaN}_{3}$ in PBS.

\section{D dSTORM Super Resolution Imaging and Reconstruction of RyRs}

Alexa Fluor 647-labeled rat ventricular cardiomyocytes were submersed in an imaging buffer containing 20\% VectaShield (H-1000, Vector Laboratories) diluted in TRIS-Glycerol (5\% v/v TRIS 1 $\mathrm{M} \mathrm{pH} 8$ in Glycerol, Sigma Aldrich). This composition has been previously shown to produce comparable, if not superior quality dSTORM images when compared with conventional oxygen scavenging-dependent systems ${ }^{48}$.

Cardiomyocytes were imaged using the Zeiss ELYRA/LSM 710 system (Carl Zeiss; Jena, Germany). A diode laser $(150 \mathrm{~mW}, 642 \mathrm{~nm})$ illuminated the sample via a plan-apo 63x $1.4 \mathrm{NA}$ oil objective, configured in a highly inclined and laminated optical sheet (HiLo). 3D imaging was achieved utilising Phase Ramp Imaging Localization Microscopy (PRILM) technology ${ }^{49}$. Fluorescence emission $>655 \mathrm{~nm}$ was collected with an iXon 897 back-thinned EMCCD camera (Andor Technology, Belfast). A sequence of 15,000 frames was acquired for each cell at a frame exposure time of $40 \mathrm{~ms}$. Throughout image acquisition, a piezo-operated Definite Focus system was employed to autocorrect for axial drift.

Reconstruction of dSTORM data was carried out using the 'PALM Processing' module in the ZEN Black software (Zeiss). In short, an experimental 3D PSF with an axial range of $4 \mu \mathrm{m}$ was acquired using $100 \mathrm{~nm}$ TetraSpecks (T7279, Thermo Fisher Scientific). Individual single molecule events were detected by employing an 11 pixel circular mask, with a signal to background noise ratio of 6 . Drift correction was performed using a 5 segment piece-wise linear function, and a text-based points table was then generated containing the $\mathrm{x}, \mathrm{y}$ and $\mathrm{z}$ coordinates of each localization event. In order to minimize the inclusion of clusters with larger localization error, events from only the central $600 \mathrm{~nm}$ of the $4 \mu \mathrm{m}$ stack were included ${ }^{49}$. Lastly, the points table was processed via a custom-written Python script, to generate a pixel based image whereby individual events were represented with a Gaussian function 
centered at the event coordinates and a width corresponding to its lateral and axial precision values (in $\mathrm{nm})$. The resulting intensity data stack was then thresholded using the Ostsu method and output as a $600 \mathrm{~nm}$ z-stack with a voxel size of $30 \mathrm{~nm}$, so that each voxel of the resulting 3D binary mask stack contained no more than a single RyR.

\section{Quantitative Analysis of 3D RyR Cluster Characteristics}

613 3D quantification of RyR organization was performed as previously described ${ }^{5}$. In short, by combining

614 Phase Ramp Imaging Localization Microscopy (PRILM) dSTORM and fluorescent event registration,

615 we were able to discern the irregular shapes of internal RyR arrangements, and estimate RyR cluster

616 and CRU sizes. Based on mathematical modeling by Sobie et al. ${ }^{3}$, RyR clusters with edges localized

617 within $100 \mathrm{~nm}$ were assigned to the same CRU. RyR cluster density was calculated and normalized by

618 totaling the number of clusters within a $2 \times 2 \times 0.6 \mu \mathrm{m}$ volume positioned within the cell interior. The

619 volumetric density of RyRs was determined on a per cell basis by multiplying the average cluster size

620 by the cluster density.

\section{Correlative Imaging of RyRs and t-tubules for Dyad Reconstruction}

623

The Zeiss ELYRA system was also used for confocal imaging of RyRs (Alexa Fluor 647, $633 \mathrm{~nm}$ laser)

624 and t-tubules (Alexa Fluor 488, $488 \mathrm{~nm}$ laser) for 3D correlative reconstruction of dyads. Prior to dSTORM imaging of RyRs, a $3 \mu \mathrm{M}$ thick confocal stack centered on the same region was acquired (frame size: 1024 x 1024 pixels; pixel size: $50 \mathrm{~nm}$; z spacing: $200 \mathrm{~nm}$ per slice). Deconvolution of confocal images was performed using Huygens Essential software (SVI, The Netherlands), with a signal to noise ratio of 5. Using the confocally-imaged RyR channel as a reference, confocally measured ttubule distributions were correlated to dSTORM-derived RyR positions. This was done with a customwritten Python script which corrected for both lateral, axial, translational and scaling differences between the two imaging modalities to optimally align the dSTORM-derived RyR data with the confocal RyR data.

\section{D Geometric Rendering of RyRs and T-tubules}

To examine the arrangement of RyR clusters in relation to t-tubules, 3D volumetric geometries were constructed from the correlated images of these structures using the same approach as described in our earlier work ${ }^{5}$. Geometries were built using a custom Python script relying on NumPy $1.18^{50}$, SciPy $1.5^{51}$ and scikit-image $0.15^{52}$ for morphological manipulation.

A 3D reconstruction of the t-tubule network was created from confocal NCX1/Cav-3 imaging by first fitting the data to $10 \mathrm{~nm} \times 10 \mathrm{~nm} \times 10 \mathrm{~nm}$ voxels, binarizing using an adaptive Gaussian threshold, and then pruning small non-contiguous components $\left(<0.03 \mu \mathrm{m}^{3}\right)$. The resulting thresholded structures were skeletonized using a 3D skeletonization algorithm ${ }^{53}$, and re-dilated to form $250 \mathrm{~nm}$ wide cylindrical ttubules $^{54}$. The correlated dSTORM imaging data of RyRs were then fitted to the same voxel grid and Otsu-thresholded. The majority of the thresholded clusters directly overlapped with the defined dyadic RyR face. Due to inaccuracies in the t-tubule reconstruction as well as limited precision in the underlying confocal imaging, all clusters lying within $150 \mathrm{~nm}$ of a reconstructed t-tubule were estimated to be dyadic clusters. More distally localized RyR clusters were deemed to be non-dyadic.

To render the 3D geometric reconstructions and produce geometric meshes for use in mathematical modeling, specific locations of individual RyRs were assigned by projecting the thresholded dyadic cluster data onto a cylindrical shell surrounding the t-tubules, leaving a 1-voxel wide $(10 \mathrm{~nm})$ dyadic cleft. These overlap interfaces were then randomly filled with RyRs such that no channels were closer 
than a center-to-center distance of $30 \mathrm{~nm}$. As shown in our earlier work, this approach yields RyR numbers that are in good agreement with our experimentally-obtained estimates ${ }^{5}$.

To visualize the reconstructed geometry of t-tubules and RyRs, iso-surfaces were generated from the full geometries using the Lewiner Marching-Cubes algorithm ${ }^{55}$, smoothed using GAMer $2.0^{56}$, and finally rendered using Blender (Blender Foundation, the Netherlands).

For use in computational modeling, an SR network was heuristically added to the voxel-based reconstruction of t-tubules and RyRs. The junctional SR (jSR) terminals for each cluster were defined by iterative morphological binary dilation of the RyRs constrained to a $20 \mathrm{~nm}$ wide cylindrical shell surrounding the t-tubules. Dilation was performed with a structuring element with a square connectivity of one for nine iterations. This method was chosen because it produces a jSR that evenly surrounds the RyR channels in the cluster, defines a well constrained dyadic cleft space, and has a jSR volume on the order of 3-4 $\times 10^{-12} \mu \mathrm{l}$, which is in good agreement with values reported by electron microscopy tomography ${ }^{57}$. The non-junctional network SR (nSR) was added as a regular grid of thin structures throughout the cytosol. While the generated morphology is artificial, the created structure has a volume and surface area in agreement with $3 \mathrm{D}$ electron microscopy data ${ }^{57}$ and serves to connect the different CRUs.

For each CRU in the fully reconstructed volumetric geometry, a surrounding region measuring $1 \mu \mathrm{mx}$ $1 \mu \mathrm{m} \times 1 \mu \mathrm{m}$ was extracted, producing a set of smaller geometries representing individual CRUs for computational modeling of $\mathrm{Ca}^{2+}$ release. Roughly one thousand such CRU geometries were extracted and sorted based on the number of contained RyRs, their spatial spread (measured as the root-meansquare of their distance to the cluster center), and the number of individual clusters within the CRU. Four representative geometries were selected containing roughly the same number of RyRs, but with different degrees of compactness. These ranged from a completely solid CRU to a majorly dispersed CRU consisting of numerous, sprawled clusters (Supplementary Table 1).

\section{$\mathrm{Ca}^{2+}$ Imaging and Analysis}

684

Using an LSM 7Live confocal microscope (Zeiss), spontaneous $\mathrm{Ca}^{2+}$ sparks were recorded from quiescent cardiomyocytes loaded with fluo-4 AM (20 $\mu \mathrm{mol} / \mathrm{L}$, Molecular Probes, Oregon, USA) and superfused with Hepes-Tyrode solution containing (in mmol/L): $140 \mathrm{NaCl}, 0.5 \mathrm{MgCl}$, 5.0 HEPES, 5.5

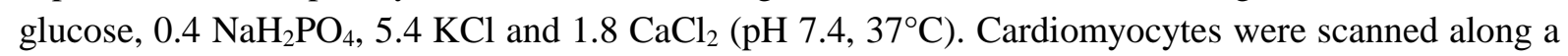
1024 pixel line drawn across the cell's longitudinal axis, at a temporal resolution of $1.5 \mathrm{~ms}$ for a total duration of $6 \mathrm{~s}$. $\mathrm{Ca}^{2+}$ sparks were analyzed using a custom program (CaSparks 1.01, D. Ursu, 2003) as previously ${ }^{25}$, with sparks defined as local increases in fluorescence intensity of at least 3 times above background $\left(\Delta F / F_{0} \geq 0.3\right)$. Spark characteristics outputted by the program included amplitude $\left(\Delta F / F_{0}\right)$, time to peak (TTP), full duration at half maximum (FDHM), and full width at half maximum (FWHM). Spark frequency was determined by normalizing spark count to the cell length and recording duration.

$\mathrm{Ca}^{2+}$ transients were similarly recorded by confocal line-scans in fluo-4 loaded cells, but during fieldstimulation via a pair of platinum electrodes ( $3 \mathrm{~ms}$ supra-threshold current pulses at $1 \mathrm{~Hz}$ ). As previously described ${ }^{8}, \mathrm{Ca}^{2+}$ release synchrony was calculated by plotting the profile of time to $50 \%$ peak fluorescence $\left(\mathrm{TTF}_{50}\right)$ across the cell and measuring the standard deviation of the values. We have termed this measure the 'dyssynchrony index'. Spontaneous $\mathrm{Ca}^{2+}$ waves were measured during pauses in the electrical excitation, and wave frequency was normalized to the recording duration. $\mathrm{SR} \mathrm{Ca}^{2+}$ content was estimated by measuring the increase in cytosolic $\mathrm{Ca}^{2+}$ fluorescence after superfusing cardiomyocytes with $10 \mathrm{mM}$ caffeine (Sigma-Aldrich). SERCA activity was estimated as the difference 
between the rate constants $(1 / \tau)$ of the decline of steady-state $\mathrm{Ca}^{2+}$ transient $(1 \mathrm{~Hz})$ and the caffeineinduced $\mathrm{Ca}^{2+}$ transient $(1 / \tau 1 \mathrm{~Hz}-1 / \tau$ caffeine $)$ acquired in the same cell ${ }^{58}$.

\section{Western Blotting}

Frozen tissue from rat left ventricles was homogenized in cold buffer $(210 \mathrm{mM}$ sucrose, $2 \mathrm{mM}$ EGTA, $40 \mathrm{mM} \mathrm{NaCl}, 30 \mathrm{mM}$ HEPES, $5 \mathrm{mM}$ EDTA) with the addition of a Complete EDTA free protease inhibitor cocktail tablet (Roche Diagnostics, Oslo, Norway) and a PhosSTOP tablet (Roche). SDS was then added to the homogenates to a final concentration of $1 \%$, and protein concentrations were quantified using a micro BCA protein assay kit (Thermo Fischer Scientific Inc., Rockford, IL). Bovine serum albumin (BSA) was used as standard protein.

Protein homogenates ( 5 or $15 \mu \mathrm{g} / \mathrm{lane}$ ) were size fractionated on $4-15 \%$ or $15 \%$ Criterion TGX gels (Biorad Laboratories, Oslo, Norway) and transferred to $0.45 \mu \mathrm{M}$ PVDF-membranes (GE Healthcare). The membranes were blocked in 5\% non-fat milk or 5\% Casein (Roche Diagnostics) in Tris-buffered saline with $0.1 \%$ Tween (TBS-T) for $1 \mathrm{hr}$ at room temperature, and then incubated with primary antibody overnight at $4{ }^{\circ} \mathrm{C}$. The following primary antibodies were employed for immunoblotting: RyR2 (1:1000) (MA3-916, Thermo Fisher Scientific), pSer2808 RyR2 (1:2500) (A010-30, Badrilla), pSer2814 RyR2 (1:2500) (A010-31, Badrilla) and GAPDH (1:500; sc-20357, Santa Cruz Biotechnology). Secondary antibodies were anti-rabbit (NA934V, GE Healthcare) or anti-mouse (NA931V, GE Healthcare). These were incubated for $1 \mathrm{hr}$ at room temperature and blots were developed using Enhanced Chemiluminescence (ECL prime, GE healthcare). Chemiluminescence signals were detected by a LAS 4000 (GE healthcare) and protein levels were quantified using ImageQuant software (GE Healthcare). Results were normalized to GAPDH and then to Sham values.

\section{Flow cytometry using Fluorescence-activated cell sorting}

In order to examine the relative phosphorylation levels of isoproterenol-treated cardiomyocytes, we employed Fluorescence-activated cell sorting. This technique has previously been shown to enable protein quantification in close correlation with results from Western blotting ${ }^{59}$. Fixed cells labelled with primary antibodies against pSer-2808 RyR2 (A010-30, Badrilla) or pSer-2814 RyR2 (A010-31, Badrilla) and secondary AlexaFluor647 antibody were placed in a suspension of PBS with 1\% BSA. Flow cytometry analysis was performed on a Sony SH800 Cell Sorter and FlowJo ${ }^{\text {TM }}$ Software (Becton, Dickinson and Company; Ashland, OR, USA) was used for analysis. Control treatments were included which contained unlabelled cells, or only secondary antibody. Voltage settings for the side, back, and forward scatter were kept constant for each experiments described. The cell suspension was analysed at a flow rate of $27 \mu \mathrm{L} / \mathrm{min}$, until $>5000$ events were captured. A two-step sorting process was employed. Intact cardiomyocytes were separated from other cell types and debris using a dual parameter dot plot for side- and forward scatter (see Supplementary Fig. 2a for representative image). Single cardiomyocytes were separated from doublets using a plot of forward scatter height and pulse width (Supplementary Fig. 2b).

Following sorting, the secondary AlexaFluor647 antibody (A-21237, Thermo Fisher Scientific) in the single cardiomyocyte fraction was excited with the $638 \mathrm{~nm}$ laser and emission detected by a photomultiplier tube with a 665/30 Band pass filter. Data are presented as detected fluorescence intensity as a product of cell size. Confirmation of cell viability was performed using LIVE/DEAD ${ }^{\mathrm{TM}}$ a 450/50 band pass filter. 


\section{Mathematical Modeling of $\mathrm{Ca}^{2+}$ Sparks}

Effects of changing CRU configuration on $\mathrm{Ca}^{2+}$ spark characteristics were interrogated using a mathematical model of $\mathrm{Ca}^{2+}$ release in the dyad. This previously described reaction-diffusion model includes independent, stochastic RyR channels coupled with deterministic $\mathrm{Ca}^{2+}$ diffusion and buffering ${ }^{11}$. The model was chosen for the current work since it can incorporate changing CRU configurations based on super-resolution imaging. The model was employed as previously described, but with minor adjustments to better align $\mathrm{Ca}^{2+}$ release and pump-leak balance behaviour with experimental data from rat and mouse ${ }^{60-62}$. Specifically, we slightly augmented $\mathrm{SR} \mathrm{Ca}^{2+}$ reuptake by increasing in the amount and density of SERCA, and modestly decreased SR volume and buffering to reduce releasable $\mathrm{Ca}^{2+}$. When combined with a slightly more sensitive $\mathrm{RyR}_{\text {model }}{ }^{63}$, these modifications yielded larger fractional SR $\mathrm{Ca}^{2+}$ release, in better agreement with experimental data. Please refer to Supplementary Tables 2 and 3 for a full list of buffering and RyR parameters.

To incorporate the effects of isoproterenol treatment on $\mathrm{Ca}^{2+}$ signaling in the $\mathrm{CRU}$, we altered two model parameters; the SR $\mathrm{Ca}^{2+}$ load was increased from $900 \mu \mathrm{M}$ at baseline to $1300 \mu \mathrm{M}$, and the RyR opening sensitivity to cytosolic $\mathrm{Ca}^{2+}$ was increased by lowering the $\mathrm{K}_{\mathrm{d}}$ open from $45 \mu \mathrm{M}$ to $25 \mu \mathrm{M}$. These two model parameters and their adjustments were constrained by comparing the amplitude and rise time of simulated sparks to experimental measurements made in control and isoproterenol treatment conditions (Supplementary Fig. 4).

\section{Analysis of Spark Simulations}

400 simulations were performed for both the baseline and isoproterenol parameter sets, with each CRU geometry. The initial conditions were otherwise identical for each simulation. A single, randomlyselected RyR in the CRU was opened, and the simulation was then allowed to progress stochastically. The probability of each RyR opening and closing was thus based on its own locally-sensed $\mathrm{Ca}^{2+}$ concentration. The simulations continued until all RyRs in the CRU had been simultaneously closed for at least $1 \mathrm{~ms}$, at which point the simulation was terminated. Thus, the full tail of the spark was not modelled out of consideration for computational efficiency.

To compare the modelled with experimentally-measured sparks, we used the average concentration of $\mathrm{Ca}^{2+}$-bound Fluo in the full cytosol of the $1 \mu \mathrm{m} \times 1 \mu \mathrm{m} \times 1 \mu \mathrm{m}$ computational domain. This measure was employed instead of more computationally expensive line scan simulations, since our previous work indicated that this simplified approach yields equal predictions of spark amplitude and time course $^{11}$.

For each simulation, we measured the peak amplitude of the Fluo signal and the rise time (TTP). Based on the measured amplitude, we then determined which simulations to consider sparks, using the same threshold as in the experimentally-measured $\mathrm{Ca}^{2+}$ sparks $\left(\Delta \mathrm{F} / \mathrm{F}_{0} \geq 0.3\right)$. For simulations where the peak amplitude was below this threshold, we defined sub-spark events as "quarks" $30\left(0.1<\Delta \mathrm{F} / \mathrm{F}_{0}<0.3\right)$, or "failed sparks" if $\mathrm{Ca}^{2+}$ release was negligible $\left(\Delta \mathrm{F} / \mathrm{F}_{0} \leq 0.1\right)$.

Based on the ratio of spark events to sub-spark events, we estimated the probability of a spontaneous opening leading to an observable spark, dubbed the "spark fidelity". As each individual simulation was an independent stochastic trial in a Bernoulli process, each simulation produced a spark with probability equal to the fidelity. To estimate the fidelity, we used the maximum-likelihood predictor of the probability, which is simply the observed ratio of successful sparks to total simulations. Extra care should be taken in calculating a confidence interval for spark fidelity, as we might expect the fidelity to 
be very close to or equal to 0 for severely fragmented clusters. We therefore opted to follow the recommendation of Brown, Cai and DasGupta ${ }^{64}$ and used the Agresti-Coull confidence interval ${ }^{65}$.

We also quantified the amount of released $\mathrm{Ca}^{2+}$ during each simulation. For simulations that were deemed to elicit observable sparks, the released $\mathrm{Ca}^{2+}$ was considered spark-mediated leak, while subspark events defined "silent" leak. Total RyR-mediated leak was calculated as the sum of the two types of events. As the mathematical model was mainly constrained using amplitude and TTP values of sparks, the leak measurements were not analyzed in absolute terms, but rather as the relative amount of spark-mediated and silent leak in the different geometry and parameter combinations.

\section{Statistical Analyses}

808 Cardiomyocytes from rats were randomly selected for analysis of RyR localisation and calcium imaging. Power analysis was performed to determine sample sizes based on known variability of measured parameters. No data were excluded from the analyses, and consistent observations were made during analyses performed on different cardiomyocytes from different hearts. Experiments involving multiple treatment groups were all carried out in the same day to ensure consistency. Statistical significance between sample means was calculated by nested ANOVA (SPSS, IBM), with Fisher's least significant difference (LSD) post-hoc comparison where appropriate. $\mathrm{P}$ values $<0.05$ were considered to be significant. All results presented are expressed as mean \pm standard error of the mean (SEM) unless otherwise stated.

817

\section{Code Availability}

819 Custom code used in this study is available at the public repository https://gitlab.com/louch-group/ryrtt-correlative-analsyis.

\section{Acknowledgements}

We thank the Section for Comparative Medicine at Oslo University Hospital Ullevål for expert animal care. This study was financially supported by the European Union's Horizon 2020 research and innovation progamme (Consolidator grant, W.E.L.) under grant agreement No. 647714, the Norwegian Research Council (X.S., W.E.L.), and The Norwegian Association for Public Health (W.E.L.). This work used the Oakforest-PACS supercomputer system provided by The University of Tokyo through Joint Usage/Research Center for Interdisciplinary Large-scale Information Infrastructures and High Performance Computing Infrastructure in Japan (Project IDs: JHPCN-jh180024, JHPCN-jh190040 \& JHPCN-jh200036).

\section{Author Contributions} and W.E.L wrote the manuscript. X.S., A.B-D., T.R.K., E.S.N., Y.H., A.P.Q., E.K.S.E. and I.S. performed the experiments. X.S., J.vdB., A.B-D., M.L., E.K.S.E. and I.S. analyzed data. X.S., J.vdB., M.L., I.S., X.H.T.W., A.G.E., C.S. and W.E.L discussed data and gave conceptual advice. All authors critically reviewed the manuscript.

\section{Competing Financial Interests}




\section{References}

1. Bers, D.M. Excitation-contraction coupling and cardiac contractile force., (Kluwer Academic, Dordrecht, Netherlands, 2001).

2. Baddeley, D., et al. Optical single-channel resolution imaging of the ryanodine receptor distribution in rat cardiac myocytes. Proceedings of the National Academy of Sciences of the United States of America 106, 22275-22280 (2009).

3. Sobie, E.A., et al. The $\mathrm{Ca}^{2+}$ leak paradox and "rogue ryanodine receptors": $\mathrm{SR} \mathrm{Ca}^{2+}$ efflux theory and practice. Progress in biophysics and molecular biology 90, 172-185 (2006).

4. Cheng, H., Lederer, W. \& Cannell, M. Calcium sparks: elementary events underlying excitation-contraction coupling in heart muscle. Science 262, 740-744 (1993).

5. Shen, X., et al. 3D dSTORM imaging reveals novel detail of ryanodine receptor localization in rat cardiac myocytes. 597, 399-418 (2019).

6. Jayasinghe, I., et al. True Molecular Scale Visualization of Variable Clustering Properties of Ryanodine Receptors. Cell Reports 22, 557-567 (2018).

7. Asghari, P., et al. Nonuniform and variable arrangements of ryanodine receptors within mammalian ventricular couplons. Circulation Research 115, 252-262 (2014).

8. Louch, W.E., et al. T-tubule disorganization and reduced synchrony of $\mathrm{Ca}^{2+}$ release in murine cardiomyocytes following myocardial infarction. The Journal of Physiology 574, 519-533 (2006).

9. Fowler, E.D., et al. Arrhythmogenic late $\mathrm{Ca}^{2+}$ sparks in failing heart cells and their control by action potential configuration. Proceedings of the National Academy of Sciences 117, 2687$2692(2020)$.

10. Orchard, C.H., Bryant, S.M. \& James, A.F. Do t-tubules play a role in arrhythmogenesis in cardiac ventricular myocytes? The Journal of physiology 591, 4141-4147 (2013).

11. Kolstad, T.R., et al. Ryanodine receptor dispersion disrupts $\mathrm{Ca}^{2+}$ release in failing cardiac myocytes. eLife 7, e39427 (2018).

12. Macquaide, N., et al. Ryanodine receptor cluster fragmentation and redistribution in persistent atrial fibrillation enhance calcium release. Cardiovascular Research 108, 387-398 (2015).

13. Sheard, T.M.D., et al. Three-Dimensional and Chemical Mapping of Intracellular Signaling Nanodomains in Health and Disease with Enhanced Expansion Microscopy. ACS nano 13, 2143-2157 (2019).

14. Asghari, P., et al. Cardiac ryanodine receptor distribution is dynamic and changed by auxiliary proteins and post-translational modification. eLife 9, e51602 (2020).

15. Ling, H., et al. Requirement for $\mathrm{Ca}^{2+} /$ calmodulin-dependent kinase II in the transition from pressure overload-induced cardiac hypertrophy to heart failure in mice. J Clin Invest 119, 12301240 (2009).

16. Zhang, T., et al. The $\delta \mathrm{C}$ Isoform of CaMKII Is Activated in Cardiac Hypertrophy and Induces Dilated Cardiomyopathy and Heart Failure. Circulation Research 92, 912-919 (2003).

17. Marx, S.O., et al. PKA Phosphorylation Dissociates FKBP12.6 from the Calcium Release Channel (Ryanodine Receptor): Defective Regulation in Failing Hearts. Cell 101, 365-376 (2000).

18. Grimm, M. \& Brown, J.H. Beta-adrenergic receptor signaling in the heart: role of CaMKII. Journal of molecular and cellular cardiology 48, 322-330 (2010).

19. Grimm, M., et al. CaMKII $\delta$ mediates $\beta$-adrenergic effects on RyR2 phosphorylation and SR $\mathrm{Ca}^{2+}$ leak and the pathophysiological response to chronic $\beta$-adrenergic stimulation. Journal of molecular and cellular cardiology 85, 282-291 (2015).

20. Ferrero, P., et al. $\mathrm{Ca}^{2+} /$ calmodulin kinase II increases ryanodine binding and $\mathrm{Ca}^{2+}$-induced sarcoplasmic reticulum $\mathrm{Ca}^{2+}$ release kinetics during beta-adrenergic stimulation. Journal of molecular and cellular cardiology 43, 281-291 (2007).

21. Pereira, L., et al. Epac2 mediates cardiac $\beta 1$-adrenergic-dependent sarcoplasmic reticulum $\mathrm{Ca}^{2+}$ leak and arrhythmia. Circulation 127, 913-922 (2013). 
22. Øyehaug, L., et al. Synchrony of Cardiomyocyte $\mathrm{Ca}^{2+}$ Release is Controlled by t-tubule Organization, SR $\mathrm{Ca}^{2+}$ Content, and Ryanodine Receptor $\mathrm{Ca}^{2+}$ Sensitivity. Biophysical Journal 104, 1685-1697 (2013).

23. Christensen, A.E., et al. cAMP analog mapping of Epac1 and cAMP kinase: Discriminating analogs demonstrate that Epac and cAMP kinase act synergistically to promote PC-12 cell neurite extension. Journal of Biological Chemistry 278, 35394-35402 (2003).

24. Szabo-Fresnais, N., Lefebvre, F., Germain, A., Fischmeister, R. \& Pomérance, M. A new regulation of IL- 6 production in adult cardiomyocytes by $\beta$-adrenergic and IL-1 $\beta$ receptors and induction of cellular hypertrophy by IL-6 trans-signalling. Cellular Signalling 22, 1143-1152 (2010).

25. Louch, W.E., et al. Slow $\mathrm{Ca}^{2+}$ sparks de-synchronize $\mathrm{Ca}^{2+}$ release in failing cardiomyocytes: Evidence for altered configuration of $\mathrm{Ca}^{2+}$ release units? Journal of Molecular and Cellular Cardiology 58, 41-52 (2013).

26. Tanaka, H., et al. Two-Dimensional Millisecond Analysis of Intracellular $\mathrm{Ca}^{2+}$ Sparks in Cardiac Myocytes by Rapid Scanning Confocal Microscopy: Increase in Amplitude by Isoproterenol. Biochemical and Biophysical Research Communications 233, 413-418 (1997).

27. Viatchenko-Karpinski, S. \& Györke, S. Modulation of the $\mathrm{Ca}^{2+}$-induced $\mathrm{Ca}^{2+}$ release cascade by $\beta$-adrenergic stimulation in rat ventricular myocytes. The Journal of Physiology 533, 837848 (2001).

28. Laasmaa, M., Karro, N., Birkedal, R. \& Vendelin, M. IOCBIO Sparks detection and analysis software. PeerJ 7, e6652 (2019).

29. Brette, F., Sallé, L. \& Orchard, C.H. Differential Modulation of L-type Ca ${ }^{2+}$ Current by SR $\mathrm{Ca}^{2+}$ Release at the T-Tubules and Surface Membrane of Rat Ventricular Myocytes. Circulation Research 95, e1-e7 (2004).

30. Brochet, D.X.P., Xie, W., Yang, D., Cheng, H. \& Lederer, W.J. Quarky calcium release in the heart. Circulation research 108, 210-218 (2011).

31. Frisk, M., et al. Elevated ventricular wall stress disrupts cardiomyocyte t-tubule structure and calcium homeostasis. Cardiovascular Research 112, 443-451 (2016).

32. Song, L.-S., et al. Orphaned ryanodine receptors in the failing heart. Proceedings of the National Academy of Sciences of the United States of America 103, 4305-4310 (2006).

33. Zima, A.V., Picht, E., Bers, D.M. \& Blatter, L.A. Termination of cardiac $\mathrm{Ca}^{2+}$ sparks: role of intra-SR $\left[\mathrm{Ca}^{2+}\right]$, release flux, and intra-SR $\mathrm{Ca}^{2+}$ diffusion. Circulation research 103, e105-e115 (2008).

34. Walker, Mark A., et al. Superresolution Modeling of Calcium Release in the Heart. Biophysical Journal 107, 3018-3029 (2014).

35. Galice, S., Xie, Y., Yang, Y., Sato, D. \& Bers, D.M. Size Matters: Ryanodine Receptor Cluster Size Affects Arrhythmogenic Sarcoplasmic Reticulum Calcium Release. J Am Heart Assoc 7, e008724 (2018).

36. Cabra, V., Murayama, T. \& Samsó, M. Ultrastructural Analysis of Self-Associated RyR2s. Biophysical journal 110, 2651-2662 (2016).

37. Kayki-Mutlu, G. \& Koch, W.J. Nitric Oxide and S-Nitrosylation in Cardiac Regulation: G Protein-Coupled Receptor Kinase-2 and $\beta$-Arrestins as Targets. Int J Mol Sci 22, 521 (2021).

38. Wang, Q., Wang, W., Wang, G., Rodney, G.G. \& Wehrens, X.H.T. Crosstalk between RyR2 oxidation and phosphorylation contributes to cardiac dysfunction in mice with Duchenne muscular dystrophy. Journal of molecular and cellular cardiology 89, 177-184 (2015).

39. Haji-Ghassemi, O., Yuchi, Z. \& Van Petegem, F. The Cardiac Ryanodine Receptor Phosphorylation Hotspot Embraces PKA in a Phosphorylation-Dependent Manner. Molecular Cell 75, 39-52.e34 (2019).

40. Xiao, B., et al. Characterization of a Novel PKA Phosphorylation Site, Serine-2030, Reveals No PKA Hyperphosphorylation of the Cardiac Ryanodine Receptor in Canine Heart Failure. Circulation Research 96, 847-855 (2005).

41. Fernández-Velasco, M., et al. Increased Ca2+ sensitivity of the ryanodine receptor mutant RyR2R4496C underlies catecholaminergic polymorphic ventricular tachycardia. Circulation research 104, 201-209 (2009). 
42. Terentyev, D., et al. Abnormal Interactions of Calsequestrin With the Ryanodine Receptor Calcium Release Channel Complex Linked to Exercise-Induced Sudden Cardiac Death. Circulation Research 98, 1151-1158 (2006).

43. Song, L.-S., et al. Paradoxical Cellular $\mathrm{Ca}^{2+}$ Signaling in Severe but Compensated Canine Left Ventricular Hypertrophy. Circulation Research 97, 457-464 (2005).

44. Chelu, M.G., et al. Calmodulin kinase II-mediated sarcoplasmic reticulum $\mathrm{Ca}^{2+}$ leak promotes atrial fibrillation in mice. J Clin Invest 119, 1940-1951 (2009).

45. Lunde, I.G., et al. Cardiac O-GlcNAc signaling is increased in hypertrophy and heart failure. Physiological Genomics 44, 162-172 (2012).

46. Sjaastad, I., Sejersted, O.M., Ilebekk, A. \& Bjørnerheim, R. Echocardiographic criteria for detection of postinfarction congestive heart failure in rats. Journal of Applied Physiology 89, 1445-1454 (2000).

47. Hodne, K., Lipsett, D.B. \& Louch, W.E. Gene Transfer in Isolated Adult Cardiomyocytes. in Cardiac Gene Therapy: Methods and Protocols (ed. Ishikawa, K.) 169-182 (Springer New York, New York, NY, 2017).

48. Olivier, N., Keller, D., Rajan, V.S., Gönczy, P. \& Manley, S. Simple buffers for 3D STORM microscopy. Biomedical Optics Express 4, 885-899 (2013).

49. Baddeley, D., Cannell, M.B. \& Soeller, C. Three-dimensional sub-100 nm super-resolution imaging of biological samples using a phase ramp in the objective pupil. Nano Research 4, 589598 (2011).

50. Walt, S.v.d., Colbert, S.C. \& Varoquaux, G. The NumPy array: a structure for efficient numerical computation. Computing in science \& engineering 13, 22-30 (2011).

51. Virtanen, P., et al. SciPy 1.0: fundamental algorithms for scientific computing in Python. Nature methods 17, 261-272 (2020).

52. Van der Walt, S., et al. scikit-image: image processing in Python. PeerJ 2, e453 (2014).

53. Lee, T.-C., Kashyap, R.L. \& Chu, C.-N. Building skeleton models via 3-D medial surface axis thinning algorithms. CVGIP: Graphical Models and Image Processing 56, $462-478$ (1994).

54. Soeller, C. \& Cannell, M.B. Examination of the Transverse Tubular System in Living Cardiac Rat Myocytes by 2-Photon Microscopy and Digital Image-Processing Techniques. Circulation Research 84, 266-275 (1999).

55. Lewiner, T., Lopes, H., Vieira, A.W. \& Tavares, G. Efficient Implementation of Marching Cubes' Cases with Topological Guarantees. Journal of Graphics Tools 8, 1-15 (2003).

56. Lee, C.T., et al. 3D mesh processing using GAMer 2 to enable reaction-diffusion simulations in realistic cellular geometries. PLOS Computational Biology 16, e1007756 (2020).

57. Hake, J., et al. Modelling cardiac calcium sparks in a three-dimensional reconstruction of a calcium release unit. J Physiol 590, 4403-4422 (2012).

58. Mørk, H.K., Sjaastad, I., Sejersted, O.M. \& Louch, W.E. Slowing of cardiomyocyte $\mathrm{Ca}^{2+}$ release and contraction during heart failure progression in postinfarction mice. American Journal of Physiology - Heart and Circulatory Physiology 296, H1069-H1079 (2009).

59. Krutzik, P.O. \& Nolan, G.P. Intracellular phospho-protein staining techniques for flow cytometry: monitoring single cell signaling events. Cytometry A 55, 61-70 (2003).

60. Shannon, T.R., Ginsburg, K.S. \& Bers, D.M. Quantitative Assessment of the SR Ca ${ }^{2+}$ LeakLoad Relationship. Circulation Research 91, 594-600 (2002).

61. Santiago, D.J., Ríos, E. \& Shannon, T.R. Isoproterenol increases the fraction of sparkdependent RyR-mediated leak in ventricular myocytes. Biophysical journal 104, 976-985 (2013).

62. Mohamed, B.A., et al. Sarcoplasmic reticulum calcium leak contributes to arrhythmia but not to heart failure progression. Science Translational Medicine 10, eaan0724 (2018).

63. Laver, D.R., Kong, C.H.T. \& Cannell, M.B. Modelling Calcium-Induced-Calcium-Release from Measurements of RyR Gating. Biophysical Journal 112, 540A-541A (2017).

64. Brown, L.D., Cai, T.T. \& DasGupta, A. Interval Estimation for a Binomial Proportion. Statistical Science 16, 101-117 (2001).

65. Agresti, A. \& Coull, B.A. Approximate is Better than "Exact" for Interval Estimation of Binomial Proportions. The American Statistician 52, 119-126 (1998). 


\section{SUPPLEMENTARY MATERIAL}

\section{Prolonged $\beta$-Adrenergic Stimulation Disperses Ryanodine Receptor Clusters in Cardiomyocytes: Implications for Heart Failure}

by Xin Shen and colleagues

This PDF file includes:

Page

\section{Supplementary Tables}

Table 1 Definition of Modeled RyR Cluster Geometries 2

Table 2 Model parameters for $\mathrm{Ca}^{2+}$ buffering 2

Table 3 RyR model parameters 2

Table $4 \quad$ Cardiac parameters from post-myocardial infarction (MI) rats with HF and Shamoperated controls

\section{Supplementary Figures}

Figure $1 \quad \beta$-AR stimulation does not influence RyR organization on the cell surface 4

Figure 2 Quantification of RyR phosphorylation by flow cytometry 5

Figure 3 Isoproterenol elicits a biphasic increase in SR Ca ${ }^{2+}$ content 6

Figure $4 \quad$ Comparison of experimentally-measured and simulated Ca ${ }^{2+}$ sparks 7

Figure $5 \quad$ Model behavior at different SR loads 8

Figure $6 \quad \mathrm{Ca}^{2+}$ transients in Sham and HF myocytes 9 
2

3

4

21

22

23

\begin{tabular}{|l|l|l|l|}
\hline Geometry Definition & № of RyRs & Spatial Spread & Clusters (№ of RyRs / cluster) \\
\hline Solid & 35 & $91.2 \mathrm{~nm}$ & $1(35)$ \\
\hline Minorly Fragmented & 36 & $114.7 \mathrm{~nm}$ & $4(22,9,4,1)$ \\
\hline Moderately Fragmented & 32 & $135.4 \mathrm{~nm}$ & $7(16,4,4,3,2,2,1)$ \\
\hline Majorly Fragmented & 34 & $174.4 \mathrm{~nm}$ & $5(12,10,5,4,3)$ \\
\hline
\end{tabular}

Supplementary Table 1. Definition of Modeled RyR Cluster Geometries. From a large number of automatically-generated 3D CRU geometries, four were selected to be used in mathematical modeling of $\mathrm{Ca}^{2+}$ release. These were chosen to represent the range of fragmentation observed experimentally, while ensuring that each CRU contained roughly the same number of RyRs. Characteristics for each CRU are listed. Spatial spread was calculated as the root mean square distance of RyRs to the CRU center.

\begin{tabular}{|c|c|c|c|c|}
\hline & $\boldsymbol{\sigma}\left(\boldsymbol{\mu \mathbf { m } ^ { 2 }} \mathbf{~} \mathbf{s}\right)$ & $\boldsymbol{B}_{\text {tot }}$ & $\boldsymbol{k}_{\text {on }}\left(\mathbf{m s}^{-\mathbf{1}}\right)$ & $\boldsymbol{k}_{\text {off }}\left(\mathbf{m s}^{-\mathbf{1}}\right)$ \\
\hline Calmodulin & 22 & $24 \mu \mathrm{M}$ & 0.034 & 0.238 \\
\hline ATP & 140 & $455 \mu \mathrm{M}$ & 0.255 & 45 \\
\hline Fluo & 20 & $50 \mu \mathrm{M}$ & 0.08 & 0.09 \\
\hline Troponin & 0 & $70 \mu \mathrm{M}$ & 0.0327 & 0.0196 \\
\hline Calsequestrin & 0 & $16 \mathrm{mM}$ & 0.102 & 65 \\
\hline
\end{tabular}

\section{Supplementary Table 2. Model parameters for $\mathrm{Ca}^{2+}$ buffering.}

\begin{tabular}{|c|c|c|c|c|}
\hline Rates & $k_{\min }\left(\mathrm{ms}^{-1}\right)$ & $k_{\max }\left(\mathrm{ms}^{-1}\right)$ & $\mathrm{K}_{\mathrm{d}}(\mu \mathrm{M})$ & $\mathrm{n}$ \\
\hline+ & $5 \times 10^{-6}$ & 0.9 & 45 or $25^{*}$ & 2.7 \\
\hline- & 0.9 & 3 & 45 & -5 \\
\hline Unitary Current & $0.235 \mathrm{pA}$ & \multicolumn{3}{|l}{} \\
\hline
\end{tabular}

\section{Supplementary Table 3. RyR model parameters.}


25

26

27

28

29

30

31

32

\begin{tabular}{|l|c|c|}
\hline Parameter & Sham $(\mathbf{n = 1 4 )}$ & MI (n=12) \\
\hline Infarct size $(\%)$ & & $59.9 \pm 2.1$ \\
\hline End-diastolic volume $(\mathrm{mL})$ & $0.33 \pm 0.01$ & $0.03 \pm 0.004^{*}$ \\
\hline End-systolic volume $(\mathrm{mL})$ & $0.09 \pm 0.01$ & $0.02 \pm 0.003^{*}$ \\
\hline Stroke volume $(\mathrm{mL})$ & $0.24 \pm 0.01$ & $0.01 \pm 0.001^{*}$ \\
\hline Ejection fraction & $0.73 \pm 0.02$ & $0.35 \pm 0.02^{*}$ \\
\hline
\end{tabular}

Supplementary Table 4. Cardiac parameters from post-myocardial infarction (MI) rats with HF and Sham-operated controls. HF development was examined by CINE MRI assessment, based on established criteria ${ }^{20}$. Infarct size is percentage of left ventricular free wall (Sham: $n_{\text {heart }}=14, \mathrm{HF}: \mathrm{n}_{\text {heart }}=12 ;{ }^{*} \mathrm{p}<0.05$ ). 

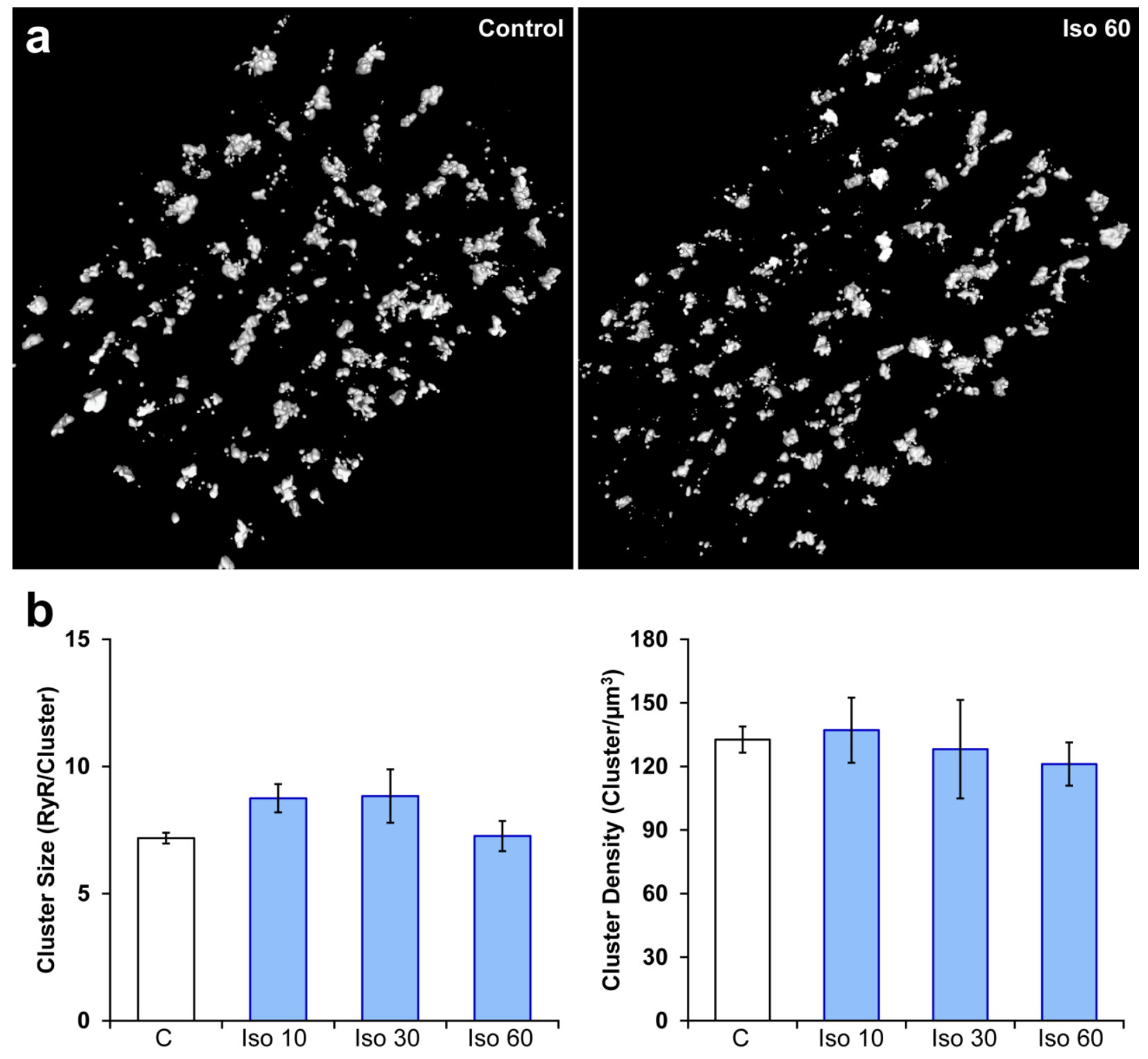

Supplementary Figure 1: $\boldsymbol{\beta}$-AR stimulation does not influence RyR organization on the cell surface.

38 Representative images (a) and mean data (b) indicate that, in contrast to internal RyRs (Fig. 1), RyR clusters

39 remain intact during prolonged $\beta$-AR stimulation. (Control: $n_{\text {cells }}=32, n_{\text {hearts }}=3$; Iso $10: n_{\text {cells }}=14, n_{\text {hearts }}=$ 404 ; Iso $30: \mathrm{n}_{\text {cells }}=12, \mathrm{n}_{\text {hearts }}=4$; Iso 60 : $\mathrm{n}_{\text {cells }}=24, \mathrm{n}_{\text {hearts }}=4$ ). 
a

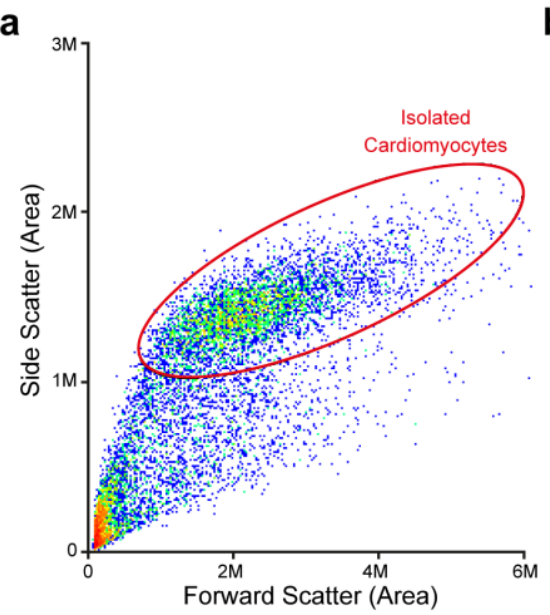

b

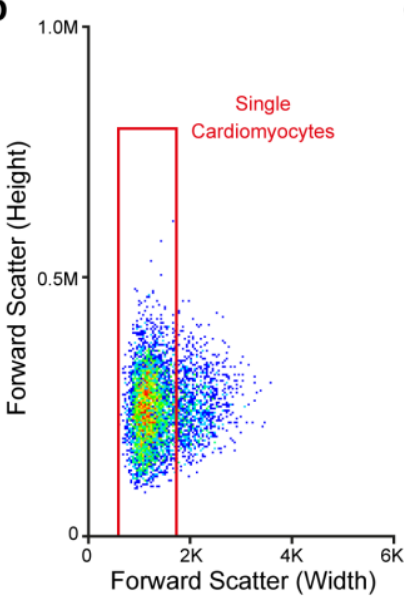

C

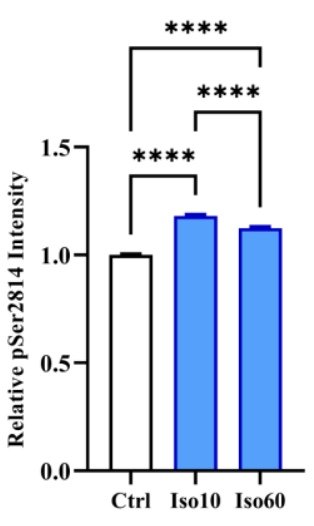

d

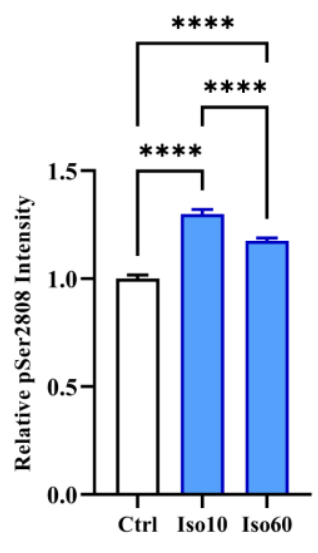

Supplementary Figure 2: Quantification of RyR phosphorylation by flow cytometry. Cardiomyocytes were separated from other cell types and debris using a dual parameter dot plot for side and forward scatter (a), and the single cardiomyocyte fraction was identified using a plot of forward scatter height and pulse width (b). (c) Fluorescent detection of RyR phosphorylation at ser-2814 revealed increased phosphorylation during $1 \mathrm{~h}$ isoproterenol treatment period, which was most prominent at the early time point examined (Control: $n_{\text {cells }}=7140$, Iso 10: $n_{\text {cells }}=6251$,; Iso 60: $n_{\text {cells }}=5253 ; n_{\text {hearts }}=2$ ). (d) RyR phosphorylation at ser-2808 also exhibited a bell-shaped response to isoproterenol treatment, but remained increased after $1 \mathrm{~h}$ of treatment. (Control: $\mathrm{n}_{\text {cells }}=742$, Iso 10: $\mathrm{n}_{\text {cells }}=803$,; Iso 60: $\mathrm{n}_{\text {cells }}=1630$; $\left.\mathrm{n}_{\text {hearts }}=1\right)$. 
a

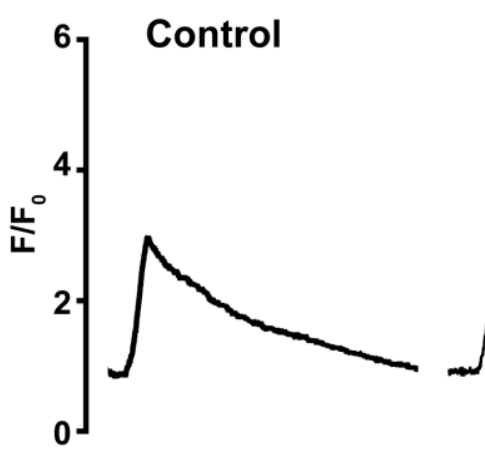

Iso 10

Iso 60
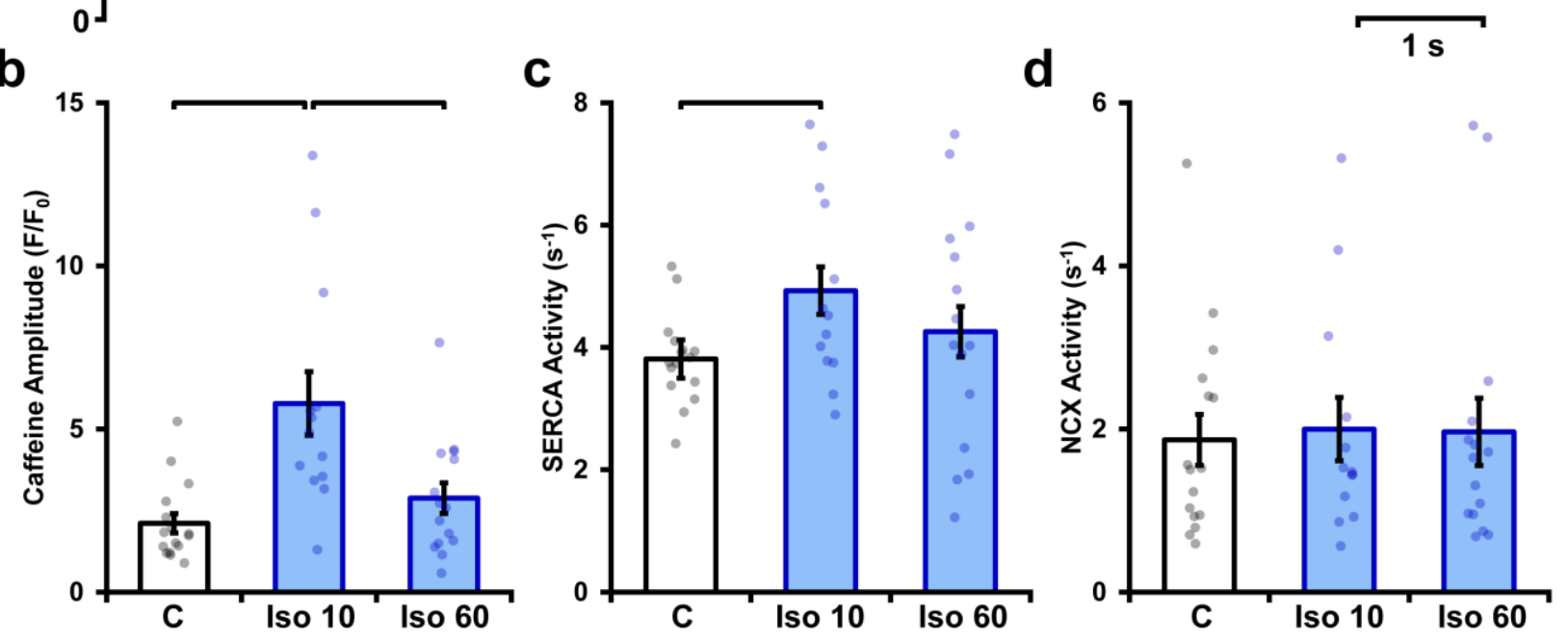

Supplementary Figure 3: Isoproterenol elicits a biphasic increase in $\mathrm{SR} \mathrm{Ca}^{2+}$ content.

$\mathrm{SR} \mathrm{Ca}^{2+}$ content was estimated by rapidly changing the superfusate to one containing $10 \mathrm{mmol} / \mathrm{L}$ caffeine, and measuring the amplitude of the elicited $\mathrm{Ca}^{2+}$ release. Representative recordings (a) and mean data (b) show that a marked increase in SR content during early isoproterenol treatment was reversed with continued exposure. SERCA and NCX activity ( $c$ and $\mathbf{d}$, respectively) were estimated based on fits of the decays of caffeine- and electrically-elicted $\mathrm{Ca}^{2+}$ transients (see methods). (Control: $\mathrm{n}_{\text {cells }}=$ 

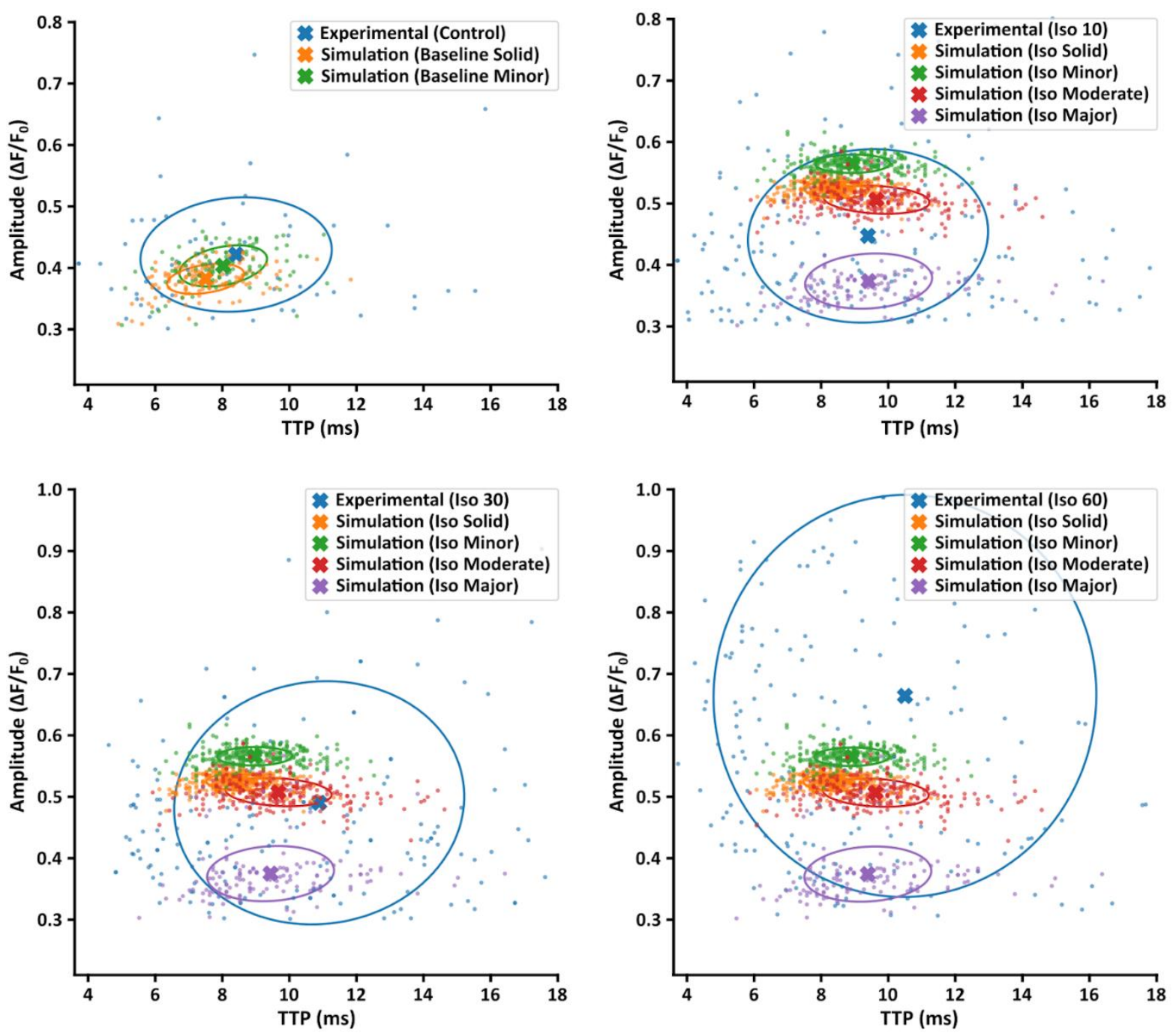

Amplitude and time to peak (TTP) values for experimentally measured $\mathrm{Ca}^{2+}$ sparks are plotted under control conditions and the three time points of isoproterenol stimulation (10, 30, $60 \mathrm{~min})$. Comparison is made with simulated sparks generated by the four modelled CRU geometries under baseline conditions, and under simulated isoproterenol conditions including increased RyR release flux and $\mathrm{Ca}^{2+}$ sensitivity. Note that the simulated sparks shown are identical in the three latter plots. Crosses indicate population means and circles show the covariance ellipse at 1 standard deviation. 

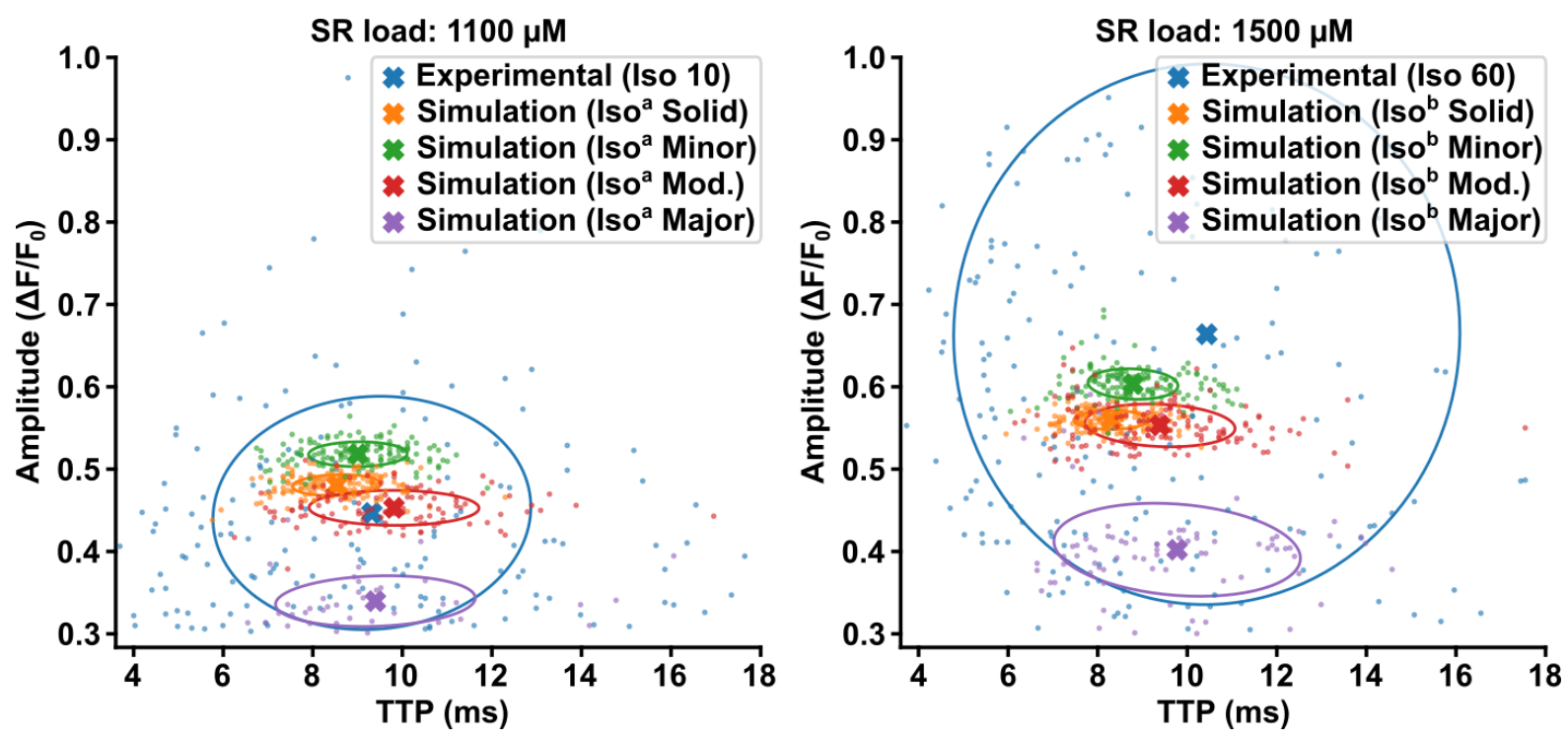

Supplementary Figure 5: Model behavior at different SR loads. To test model behavior, we perturbed the SR load parameter in the isoproterenol model while maintaining RyR $\mathrm{Ca}^{2+}$ sensitivity. We compared the standard isoproterenol model (ie. SR load $=1300 \mu \mathrm{M}$, see Supplementary Fig. 2) with SR content reduced to $1100 \mu \mathrm{M}$ (left panel, "Iso"), or increased to $1500 \mu \mathrm{M}$ (right panel, "Iso ${ }^{\mathrm{b}}$ "). These perturbations had little effect on spark TTP, with similar trends observed across the modeled CRU geometries. Spark amplitude, on the other hand, was observed to be markedly sensitive to SR load perturbation. Results are plotted alongside experimental data recorded at $10 \mathrm{~min}$ or $60 \mathrm{~min}$ following isoproterenol treatment (left

114 and right panels, respectively). 

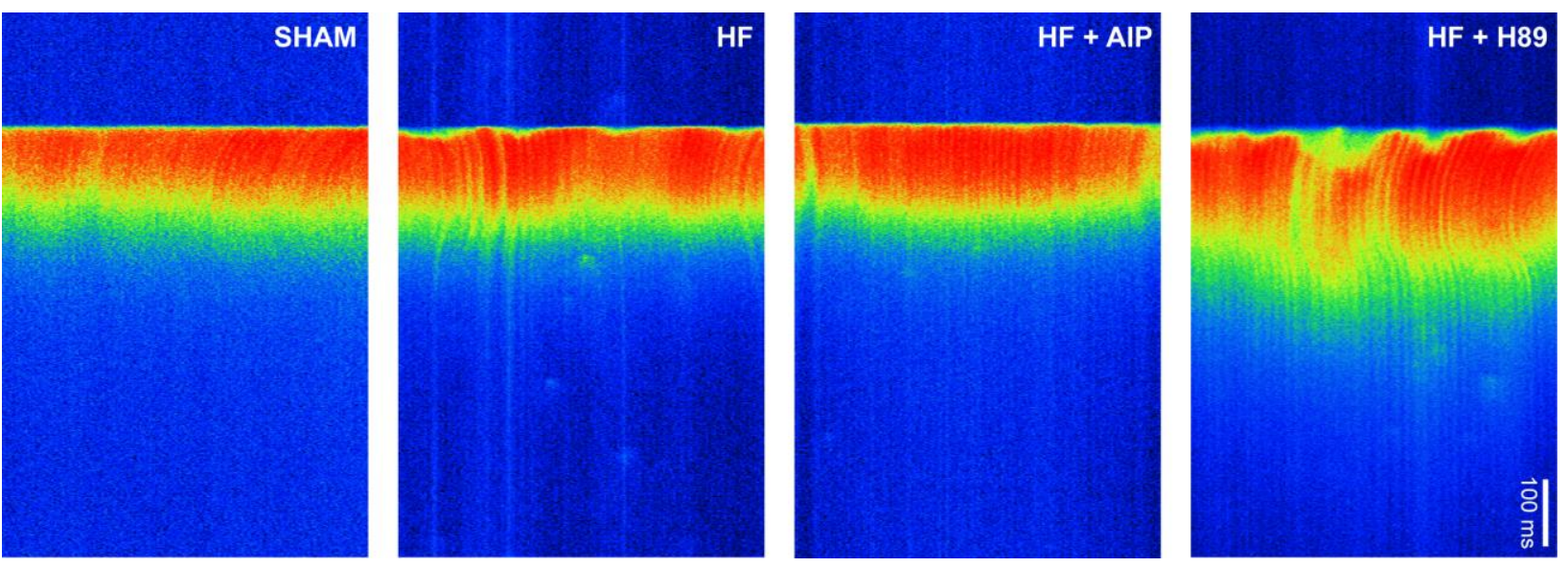

118 Supplementary Figure 6. $\mathrm{Ca}^{2+}$ transients in Sham and HF myocytes. Representative linescan images 119 illustrating the last in a series of electrically-paced $\mathrm{Ca}^{2+}$ transients. Dyssynchronous and slowed $\mathrm{Ca}^{2+}$ 120 release observed in HF myocytes was reversed by treatment with AIP but not with H89. See Fig. $6 \mathrm{~m}-\mathrm{O}$ 121 for mean measurements. 